\title{
Estabilidade assintótica de uma classe de equações quasilineares viscoelásticas com história
}

\author{
Rawlilson de Oliveira Araújo
}





\title{
Estabilidade assintótica de uma classe de equações quasilineares viscoelásticas com história ${ }^{1}$
}

\author{
Rawlilson de Oliveira Araújo
}

Orientador: Prof. Dr. Ma To Fu

Tese apresentada ao Instituto de Ciências Matemáticas e de Computação - ICMC-USP, como parte dos requisitos para obtenção do título de Doutor em Ciências - Matemática. VERSÃO REVISADA.

USP - São Carlos

Outubro de 2013

\footnotetext{
${ }^{1}$ Este trabalho teve apoio financeiro da CAPES sob o processo DS-6715832/D de 03/2009 à 02/2013
} 
Ficha catalográfica elaborada pela Biblioteca Prof. Achille Bassi e Seção Técnica de Informática, ICMC/USP, com os dados fornecidos pelo(a) autor(a)

Araújo, Rawlilson de Oliveira
Estabilidade assintótica de uma classe de equações
quasilineares viscoelásticas com história /
Rawlilson de Oliveira Araújo; orientador Ma To Fu. -
- São Carlos, 2013.
65 p.
Tese (Doutorado - Programa de Pós-Graduação em
Matemática) -- Instituto de Ciências Matemáticas e
de Computação, Universidade de São Paulo, 2013.
1. Equações diferenciais parciais. 2. equações da
onda. memória. 4. atratores globais. 5. atratores
exponenciais. I. Fu, Ma To, orient. II. Título.




\section{Agradecimentos}

“Que darei eu ao SENHOR por todos os benefícios que me tem feito?” S1 116.12.

Agradeço ao meu Senhor, pois me amou primeiro, conforme Jo 3.16, nunca me desampara e usa pessoas para me abençoar. Muito obrigado meu Deus!

À toda minha querida família que sempre me ajudou e me incentivou a estudar.

Aos meus queridos amigos e irmãos em Cristo da Assembleia de Deus - Ministério do Belém - São Carlos/SP.

Aos meus amigos e companheiros de Graduação, Mestrado e Doutorado, em especial ao Alisson Rafael A. Barbosa.

A todos os professores da UFRN, UFCG e USP/ICMC, em especial ao Dr. André Gustavo C. Pereira, ao Dr. Claudianor O. Alves, ao Dr. Marco Aurélio S. Souto, a Dra. Marcia Cristina A. B. Federson e ao Dr. Alexandre N. de Carvalho.

Ao Dr. Marcio Antonio J. Silva que me ajudou no capítulo final deste trabalho.

Ao Dr. Maurício Luciano Pelicer, ao Dr. Yuming Qin, ao grupo de Sistemas Dinâmicos Não-Lineares e ao grupo de Equações Diferenciais: Análise Matemática e Aplicações.

Ao meu orientador e amigo Dr. Ma To Fu pela orientação, conselhos e paciência.

À CAPES pelo financiamento para a conclusão deste trabalho.

Por fim, agradeço à minha amada noiva e futura esposa Sheyla Silva Marinho que sempre está comigo em todos os momentos me amando, me auxiliando e me suportando. Deus a abençoe! Te Amo! 

Este trabalho é dedicado ao estudo do comportamento a longo prazo de uma classe de equações viscoelásticas não lineares com memória, da forma

$$
\left|u_{t}\right|^{\rho} u_{t t}-\Delta u-\Delta u_{t t}+\int_{\tau}^{t} \mu(t-s) \Delta u(s) d s=h, \quad \rho>0,
$$

definida num domínio limitado de $\mathbb{R}^{N}$. Tal classe de problemas foi estudada por diversos autores desde 2001, com $\tau=0$. Os resultados existentes são principalmente devotados à existência de soluções globais, decaimento da energia, com ou sem dissipações adicionais, existência com dados pequenos, entre outros. Entretanto, a questão da unicidade de soluções e existência de atratores globais não foram discutidas em trabalhos anteriores. No presente trabalho, apresentamos resultados de unicidade e existência de atratores globais para essa classe de problemas num contexto mais geral, incluindo o caso em que $\tau=-\infty$. Além disso, incluímos um problema complementar, de quarta ordem onde estudamos a existência de atratores exponenciais.

Palavras-chave: Equações diferenciais parciais, equação da onda, viscoelasticidade, memória, unicidade, atrator global, atratores exponenciais. 

This work is concerned with the long-time behaviour of a class nonlinear viscoelastic equations of the form

$$
\left|u_{t}\right|^{\rho} u_{t t}-\Delta u-\Delta u_{t t}+\int_{-\tau}^{t} \mu(t-s) \Delta u(s) d s=h, \quad \rho>0,
$$

defined in a bounded domain of $\mathbb{R}^{N}$. Such class of problems was studied by several authors since 2001, with $\tau=0$. Existing results are mainly devoted to global existence, energy decay, with or without additional dampings, existence with small data, among others. However, uniqueness and existence of global attractors were not considered previously. In the present work, we establish some results on the uniqueness of solutions and existence of global attractors in a more general setting, including $\tau=-\infty$. In addition, we have added a second problem concerned with a fourth order equation where we study the existence of exponential attractors.

Keywords: Partial differential equations, wave equation, viscoelasticity, memory, uniqueness, global attractor, exponential attractors. 

Agradecimentos

Resumo iii

$\begin{array}{lll}\text { Abstract } & \text { v }\end{array}$

$\begin{array}{ll}\text { Introdução } & 3\end{array}$

1 Preliminares $\quad 7$

1.1 Espaços de Sobolev . . . . . . . . . . . . . . . . . . 7

1.2 Potências fracionárias de operadores lineares . . . . . . . . . . . 10

1.3 Sistemas dinâmicos e atratores globais . . . . . . . . . . . . . . 12

1.4 Dimensão fractal e atratores exponenciais . . . . . . . . . . . . . 14

1.5 Uma caracterização do atrator global . . . . . . . . . . . . . . 16

2 Um problema quasilinear viscoelástico com história $\quad 17$

2.1 O problema com história . . . . . . . . . . . . . . . 18

2.2 Primeiro teorema: boa colocação e estabilidade . . . . . . . . . . . . . 19

2.3 Prova do teorema: boa colocação . . . . . . . . . . . . . . 21

2.3.1 Existência global . . . . . . . . . . . . . . . 21

2.3.2 Dependência contínua dos dados . . . . . . . . . . . . 27

2.4 Prova do teorema: estabilidade exponencial . . . . . . . . . . . . 30 
3 Existência de um atrator global 35

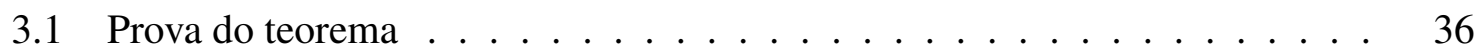

3.1 .1 Conjunto absorvente . . . . . . . . . . . . . 36

3.1 .2 Desigualdade de estabilização . . . . . . . . . . . . . 38

3.1.3 Compacidade assintótica . . . . . . . . . . . . . 41

4 Um modelo de Kirchhoff viscoelástico: atratores exponenciais 43

4.1 Introdução . . . . . . . . . . . . . . . . . . . . . . . . 43

4.2 Resultados . . . . . . . . . . . . . . . . . 46

4.3 Existência de atratores exponenciais ................ 51

4.4 Caracterização do atrator global . . . . . . . . . . . . . . . 58

$\begin{array}{ll}\text { Referências Bibliográficas } & 61\end{array}$ 



\section{Introdução}

Na presente tese, estudamos a dinâmica assintótica de dois problemas viscoelásticos não lineares que se insere no contexto das equações diferenciais hiperbólicas com termos de convolução.

O primeiro problema é baseado na equação

$$
\left|u_{t}\right|^{\rho} u_{t t}-\alpha \Delta u-\Delta u_{t t}+\int_{-\infty}^{t} \mu(t-s) \Delta u(s) d s-\gamma \Delta u_{t}+f(u)=h,
$$

definida numa região limitada $\Omega \subset \mathbb{R}^{N}$, e adicionados de dados iniciais e condição de fronteira do tipo Dirichlet. Por hipótese, tomamos $\rho>0, \gamma \geq 0$ e $\mu$ é uma função convexa decrescente. Posteriormente, a constante $\alpha>0$ será normalizada para simplificar as notações.

Do ponto de vista da modelagem matemática, a equação (1) se insere na classe

$$
g\left(u_{t}\right) u_{t t}-\Delta u-\Delta u_{t t}=0,
$$

que possui algumas aplicações em engenharias. Observamos que, mesmo quando $g\left(u_{t}\right)$ é constante, essa equação difere da equação clássica de ondas de D'Alembert por causa do termo $-\Delta u_{t t}$. De fato, no caso $g\left(u_{t}\right)=1$, a equação (2) foi usada para modelar vibrações de hastes finas (thin rods) levando em conta os efeitos da extensibilidade do material, conforme análise apresentada em Love [29], Capítulo 20. No caso em que $g\left(u_{t}\right)$ não é constante, essa equação modela vibrações de materiais cuja densidade depende da velocidade $u_{t}$. Por exemplo, segundo Cavalcanti et al [8], a equação (2) modela vibrações de uma haste fina 
que possui uma capa rígida com interior elástico levemente deformável. Por outro lado, o termo de convolução $\int_{\tau}^{t} \mu(t-s) \Delta u(s) d s$ modela propriedades viscoelásticas do material, como memória por exemplo. Assim, esta convolução é chamada de memória e a função $\mu$ é chamada de núcleo da memória. As equações viscoelásticas com memória é um tema de pesquisa bastante ativo e o leitor interessado pode consultar as referências Fabrizio \& Morro [17] e Renardy, Hrusa \& Nohel [40]. Dessa forma, o problema (1) adiciona efeitos de viscoeslasticidade ao problema (2).

No que segue, faremos uma breve revisão bibliográfica sobre o nosso problema. Acreditamos que o primeiro estudo sobre o problema (1) apareceu no trabalho de Cavalcanti, Domingos Cavalcanti \& Ferreira [8] em 2001. Eles consideraram a equação

$$
\left|u_{t}\right|^{\rho} u_{t t}-\Delta u-\Delta u_{t t}+\int_{0}^{t} g(t-s) \Delta u(s) d s-\gamma \Delta u_{t}=0
$$

definida num domínio limitado de $\mathbb{R}^{N}$, com $0<\rho<\frac{2}{N-2}$ se $N \geq 3$ e $\rho>0$ se $N=1,2$. A energia associada ao problema é definida por

$$
E(t)=\frac{1}{\rho+2}\left\|u_{t}(t)\right\|_{\rho+2}^{\rho+2}+\frac{1}{2}\|\nabla u(t)\|_{2}^{2}+\frac{1}{2}\left\|\nabla u_{t}(t)\right\|_{2}^{2} .
$$

Como estudo pioneiro, eles provaram a existência de soluções globais admitindo como hipótese $\gamma \geq 0 \mathrm{e}$

$$
-\xi_{1} g(t) \leq g^{\prime}(t) \leq-\xi_{2} g(t), \quad t \geq 0
$$

onde $\xi_{1}, \xi_{2}>0$. Esta condição diz que $g(t)$ é do tipo exponencial decrescente, o que era usual na época. Além disso, provaram que se $\gamma>0$, então a energia do sistema decai exponencialmente (quando $t \rightarrow \infty$ ). Salientamos que, quando $\rho=0$, a equação (3) se reduz à equação da onda viscoelástica usual. Nesta direção, citamos o trabalho de Cavalcanti \& Portillo Oquendo [9], que possui uma boa revisão bibliográfica e apresenta resultados essenciais.

Mais tarde, muitos outros trabalhos foram publicados sobre o problema (3). Podemos classificá-los em três grupos de interesse.

O primeiro grupo contém resultados que objetivam provar o decaimento da energia com termos de dissipação mais fracas que $-\gamma \Delta u_{t}$. Nesta direção, o trabalho mais relevante é o de Messaoudi \& Tatar [33], onde eles provam o decaimento exponencial da energia $\operatorname{com} \gamma=0$ $\mathrm{e}$

$$
g^{\prime}(t) \leq-\xi g(t)^{p}, \quad t \geq 0, \quad 1 \leq p<3 / 2
$$

Isto é, o decaimento exponencial da energia pode ser obtida apenas com a dissipação da memória (sem dissipação forte $-\gamma \Delta u_{t}$ ) com núcleo convexo decrescente, que é mais geral 
do que exponencial decrescente. Citamos também os trabalhos de Han \& Wang [22], Liu [28] e Messaoudi \& Tatar [32], que igualmente compõe esse primeiro grupo de interesse.

O segundo grupo de interesse contém resultados do tipo dados pequenos, "blow-up" em tempo finito e problemas do tipo "source-damping". Em geral, tais problemas aparecem quando adicionamos um termo de força $f(u)=k|u|^{p} u$ no lado direito da equação (3). Nesta direção, citamos os trabalho de Liu [27] e Messaoudi \& Tatar [34].

O terceiro grupo de interesse é baseado no conceito de decaimento geral em viscoelasticidade do tipo memória proposto por Messaoudi [31]. A grosso modo, supondo que o núcleo da memória satisfaz

$$
g^{\prime}(t) \leq-\xi(t) g(t) \quad t \geq 0, \quad \xi(t)>0,
$$

tenta-se obter uma taxa de decaimento da energia do tipo

$$
E(t) \leq \alpha e^{-\beta \int_{0}^{t} \xi(s) d s}, \quad t \geq 0,
$$

onde $\alpha, \beta>0$. Nesta direção, mencionamos os trabalhos de Messaoudi \& Tatar [32], Park \& Park [37], Han \& Wang [21], Liu [27] e Wu [43].

A nossa contribuição para o problema (3) é motivada pelas seguintes observações. Primeiramente, em todos os trabalhos acima citados sobre o problema em questão, a unicidade de soluções não é considerada. De fato, o termo $\left|u_{t}\right|^{\rho} u_{t t}$ parece introduzir várias dificuldades para a prova da unicidade. Além disso, do ponto de vista de sistemas dinâmicos, a existência de atratores globais também não foi considerada anteriormente. Tais propriedades são relevantes do ponto de vista físico e bastante atuais do ponto de vista da pesquisa em equações diferenciais parciais. Desta forma, o nosso objetivo nesta tese é estudar o problema (3) num contexto mais geral, com história, considerando a memória de $-\infty$ a $t$ como em (1). Então, tomando como hipótese $\rho>1$, provamos um resultado de unicidade e dependência contínua em relação aos dados iniciais. Além disso, tomando $\gamma>0$, provamos a existência de um atrator global compacto para o sistema dinâmico correspondente. Os resultados principais são provados no Teorema 2.2 (Capítulo 2) e no Teorema 3.1 (Capítulo 3). Os resultados foram recentemente publicados na referência Araújo, Ma \& Qin [4].

Sobre a metodologia, observamos que o sistema dinâmico associado ao nosso problema não possui regularidade parabólica uma vez que a equação (1) é tipicamente hiperbólica. Portanto, não podemos aplicar certos resultados clássicos da teoria dos sistemas dinâmicos de dimensão infinita. Neste caso, podemos considerar os métodos de decomposição do semigrupo (operador solução) em uma parte uniformemente estável e outra parte compacta 
(ver por exemplo Temam [42] ou Miranville \& Zelik [35]). Entretanto, esse procedimento revelou-se ser muito complexo para o nosso problema. Como alternativa, seguimos de perto a metodologia apresentada por Chueshov \& Lasiecka [11, 13] que é baseada numa desigualdade de estabilização (ver Lemma 3.4).

No estudo do primeiro problema, encontramos dificuldades em estimar a dimensão fractal do atrator global do sistema associado e em provar a existência de atratores exponenciais. Como tais conceitos fazem parte da pesquisa corrente em sistemas dinâmicos não lineares provenientes de equações diferenciais parciais, acrescentamos o estudo de um problema complementar neste trabalho.

O nosso segundo problema modela vibrações de placas viscoelásticas de quarta ordem com uma perturbação do tipo $p$-Lapaciano da forma

$$
u_{t t}+\Delta^{2} u-\Delta_{p} u-\int_{0}^{\infty} \mu(s) \Delta^{2} u(t-s) d s-\Delta u_{t}+f(u)=h
$$

definida num aberto de $\mathbb{R}^{N}$, onde

$$
\Delta_{p} u=\operatorname{div}\left(|\nabla u|^{p-2} \nabla u\right), \quad p \geq 2
$$

é o operador $p$-Laplaciano. Essa classe de problemas, sem o termo de memória, é por alguns denominada "Kirchhoff models". Ela foi estudada recentemente nos artigos de Yang [44, 45, 46, 47] e Yang \& Jin [48]. O problema também apresenta semelhanças com as equações de Kirchhoff-Boussinesq (onde $p=4$ ) estudadas por Chueshov \& Lasiecka $[10,12]$.

Por outro lado, nos trabalhos de Jorge Silva \& Ma [23, 24], tais modelos de Kirchhoff foram extendidos para o contexto da viscoelasticidade com memória. Em [23], é provada a existência de um atrator global de dimensão fractal finita para o sistema dinâmico associado ao problema (4). Entretanto, não é considerada a existência de atratores exponenciais, isto é, conjuntos compactos de dimensão fractal finita, positivamente invariantes e que atraem exponencialmente as órbitas saindo de qualquer conjunto limitado (ver Definição 1.26).

Nossa contribuição consiste em provar que o problema (4) possui um atrator exponencial. Também apresentamos uma caracterização do atrator global por meio da variedade instável proveniente do conjunto dos pontos estacionários. O resultado principal é o Teorema 4.2 (Capítulo 4). 


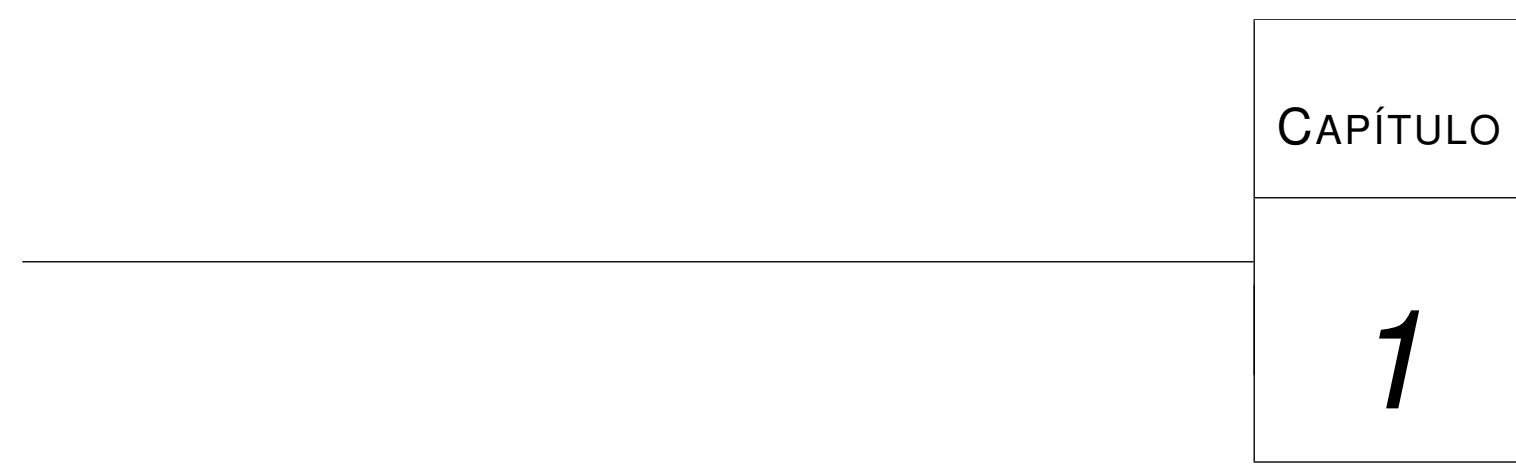

\section{Preliminares}

Neste capítulo, destacamos alguns resultados sobre espaços de funções, teoria de operadores e sistemas dinâmicos de dimensão infinita. Em relação às notações usuais no estudo das equações diferenciais não lineares, seguimos as apresentações clássicas de Evans [16] e Lions [26].

\subsection{Espaços de Sobolev}

Apresentamos aqui o teorema de imersão de Sobolev que será utilizado várias vezes. $\mathrm{O}$ leitor poderá consultar Adams \& Fournier [1], Brézis [7] para maiores informações.

Teorema 1.1 (Imersões de Sobolev). Seja $\Omega \subset \mathbb{R}^{N}$ um domínio limitado com fronteira de classe $C^{m}$.

(i) Se $m p<N$, então a seguinte inclusão é contínua

$$
W^{m, p}(\Omega) \hookrightarrow L^{q^{*}}(\Omega), \quad \text { onde } \quad \frac{1}{q^{*}}=\frac{1}{p}-\frac{m}{n} .
$$


Além disso, a inclusão é compacta para qualquer $q$, com $1 \leq q<q^{*}$.

(ii) Se $m p=N$, então a seguinte inclusão é contínua e compacta

$$
W^{m, p}(\Omega) \hookrightarrow L^{q}(\Omega), \quad \text { para todo } \quad 1 \leq q<\infty
$$

Além disso, se $p=1$ e $m=N$, então vale a mesma relação acima para $q=\infty$.

(iii) Se $k+1>m-\frac{N}{p}>k, k \in \mathbb{N}$, então escrevendo $m-\frac{N}{p}=k+\alpha$, com $0<\alpha<1$, temos que a seguinte inclusão é contínua

$$
W^{m, p}(\Omega) \hookrightarrow C^{k, \alpha}(\bar{\Omega})
$$

onde $C^{k, \alpha}(\bar{\Omega})$ representa o espaço das funções em $C^{k}(\bar{\Omega})$ cujas derivadas de ordem $k$ são $\alpha$-Hölder contínuas. Além disso, se $N=m-k-1, \alpha=1$ e $p=1$, então a inclusão vale também para $\alpha=1$, e a inclusão $W^{m, p}(\Omega) \hookrightarrow C^{k, \beta}(\bar{\Omega})$ é compacta para todo $0 \leq \beta<\alpha$.

Teorema 1.2 (Desigualdade de Gagliardo-Nirenberg). Seja $\Omega \subset \mathbb{R}^{N}$ um domínio limitado com fronteira de classe $C^{m}$ e $u \in W^{m, r}(\Omega) \cap L^{q}(\Omega)$ onde $1 \leq r, q \leq \infty$. Para qualquer inteiro $j$ com $0 \leq j<m$ e qualquer $\theta$ com $j / m \leq \theta \leq 1$, temos

$$
\left\|D^{j} u\right\|_{p} \leq C\|u\|_{m, r}^{\theta}\|u\|_{q}^{1-\theta}
$$

desde que

$$
\frac{1}{p}=\frac{j}{N}+\theta\left(\frac{1}{r}-\frac{m}{N}\right)+(1-\theta) \frac{1}{q}
$$

e $m-j-N / r$ não é um inteiro não negativo. Se $m-j-N / r$ é um inteiro não negativo, (1.1) vale $\operatorname{com} \theta=j / m$.

Teorema 1.3 (Teorema de Interpolação). Seja $\Omega \subset \mathbb{R}^{N}$ um domínio limitado com fronteira suave. Suponhamos que

$$
\begin{array}{rll}
p \leq q \leq \infty & \text { se } & m p>N, \\
p \leq q<\infty & \text { se } & m p=N, \\
p \leq q \leq \frac{N p}{N-m p} & \text { se } & m p<N .
\end{array}
$$

Então, existe uma constante $K=K(N, m, p, q, \Omega)>0$ tal que para toda $u \in W^{m, p}(\Omega)$,

$$
\|u\|_{q} \leq K\|u\|_{m, p}^{\theta}\|u\|_{p}^{1-\theta}
$$

onde $\theta=(N / m p)-(N / m q)$. 
Teorema 1.4 (Desigualdade de Poincaré). Seja $\Omega \subset \mathbb{R}^{N}$ um domínio limitado e $1 \leq p<\infty$. Então, existe uma constante $C=C(p,|\Omega|)>0$ tal que

$$
\|u\|_{p} \leq C\|\nabla u\|_{p}, \quad \forall u \in W_{0}^{1, p}(\Omega) .
$$

Definição 1.5. Seja $X$ um espaço de Banach e $1 \leq p<\infty$. Representaremos por

$$
\left(L^{p}(0, T ; X) ;\|\cdot\|_{L^{p}(0, T ; X)}\right)
$$

espaço de Banach das funções vetoriais mensuráveis $u:(0, T) \rightarrow X$, tais que $\|u(t)\|_{X}$ pertence a $L^{p}(0, T)$, munido da norma

$$
\|u\|_{L^{p}(0, T ; X)}=\left(\int_{0}^{T}\|u(t)\|_{X}^{p} d t\right)^{1 / p} .
$$

Quando $p=\infty$, representaremos por $\left(L^{\infty}(0, T ; X) ;\|\cdot\|_{L^{\infty}(0, T ; X)}\right)$ o espaço de Banach das funções vetoriais mensuráveis $u:(0, T) \longrightarrow X$, tais que $\|u(t)\|_{X}$ pertence a $L^{\infty}(0, T)$, com a norma

$$
\|u\|_{L^{\infty}(0, T ; X)}=\operatorname{supess}_{t \in(0, T)}\|u(t)\|_{X} .
$$

Temos então o Teorema de Compacidade de Simon [41, Cor. 4], que é uma extensão do Teorema de Aubin-Lions.

Teorema 1.6 (Simon). Sejam $X, B, Y$ espaços de Banach tais que $X \hookrightarrow \hookrightarrow B \hookrightarrow Y$. Suponhamos que

$$
\left(u^{n}\right) \text { é limitada em } L^{\infty}(0, T ; X) \quad e \quad\left(u_{t}^{n}\right) \text { é limitada em } L^{r}(0, T ; Y), \quad r>1 \text {. }
$$

Então, $\left(u^{n}\right)$ é relativamente compacto em $C(0, T ; B)$.

Teorema 1.7 (Desigualdade de Hölder). Sejam $1 \leq p, q \leq \infty \operatorname{com} \frac{1}{p}+\frac{1}{q}=1 e \Omega \subset \mathbb{R}^{N}$. Se $u \in L^{p}(\Omega)$ e $v \in L^{q}(\Omega)$, então $u v \in L^{1}(\Omega) e$

$$
\int_{\Omega}|u(x) v(x)| d x \leq\|u\|_{p}\|v\|_{q}
$$

Teorema 1.8 (Desigualdade de Hölder Generalizada). Sejam $1 \leq p_{1}, p_{2}, \ldots, p_{n} \leq \infty$ tais que $\frac{1}{p_{1}}+\frac{1}{p_{2}}+\cdots+\frac{1}{p_{n}}=\frac{1}{r} \leq 1$. Se $f_{i} \in L^{p_{i}}(\Omega)$ para $i=1, \ldots$, , então $f:=\prod_{i=1}^{n} f_{i} \in L^{r}(\Omega)$ $e$

$$
\|f\|_{r} \leq \prod_{i=1}^{n}\left\|f_{i}\right\|_{p_{i}}
$$


Lema 1.9 (Desigualdade de Gronwall). Sejam $\alpha \geq 0$ uma constante, $\beta \in L^{1}(a, b) e$ $\phi \in L^{\infty}(a, b)$ tais que $\beta>0$ e $\phi \geq 0$. Se

$$
\phi(t) \leq \alpha+\int_{a}^{b} \beta(s) \phi(s) d s, \quad a \leq t \leq b
$$

então

$$
\phi(t) \leq \alpha e^{\int_{a}^{b} \beta(s) d s}, \quad a \leq t \leq b .
$$

Lema 1.10 (Desigualdade de Young). Sejam $1<p, q<\infty \operatorname{com} \frac{1}{p}+\frac{1}{q}=1$. Então,

$$
a b \leq \frac{a^{p}}{p}+\frac{b^{q}}{q}, \quad \forall a, b \geq 0
$$

Lema 1.11 (Desigualdade de Young com $\epsilon$ ). Sejam $1<p, q<\infty \operatorname{com} \frac{1}{p}+\frac{1}{q}=1$ e $\epsilon>0$ qualquer. Então,

$$
a b \leq \epsilon a^{p}+C(\epsilon) b^{q}, \quad \forall a, b \geq 0,
$$

onde $C(\varepsilon)=(\epsilon p)^{-q / p} q^{-1}$.

Observação 1.12. Quando $p=q=2$, a desigualdade de Young com $\epsilon>0$ se reduz à

$$
a b \leq \epsilon a^{2}+\frac{1}{4 \epsilon} b^{2}, \quad \forall a, b \geq 0
$$

\subsection{Potências fracionárias de operadores lineares}

Nesta seção, resumimos a construção de operadores lineares não limitados associados a uma forma bilinear e comentamos um pouco sobre os operadores com potência fracionária. Para maiores informações, sugerimos os trabalhos de Temam [42] ou Kreyszig [25].

Sejam $\left(V,\|\cdot\|_{V},(\cdot, \cdot)_{V}\right)$ e $\left(H,\|\cdot\|_{H},(\cdot, \cdot)_{H}\right)$ dois espaços de Hilbert tais que $V$ é denso em $H$ e $V \hookrightarrow \hookrightarrow H$. Denotamos por $V^{\prime}$ o dual de $V$ e por $\langle\cdot, \cdot\rangle$ a dualidade entre $V^{\prime}$ e $V$. Identificando $H$ com seu dual, usando o Teorema da representação de Riesz, obtemos a seguinte cadeia de inclusões

$$
V \hookrightarrow H \cong H^{\prime} \hookrightarrow V^{\prime}
$$

Considerando uma forma bilinear contínua $a(\cdot, \cdot): V \times V \rightarrow \mathbb{R}$, definimos um operador linear $A: V \rightarrow V^{\prime}$ dado por

$$
\langle A u, v\rangle=a(u, v), \quad \forall u, v \in V
$$

e com domínio definido por

$$
D(A)=\{u \in V \mid A u \in H\} .
$$


Da teoria de análise funcional (ver Temam [42, Capítulo 2]) observamos que, se $a(\cdot, \cdot)$ é uma forma bilinear contínua, coerciva e simétrica, então o operador linear $A: D(A) \subset H \rightarrow$ $H$ é fechado, não limitado, positivo definido, autoadjunto e é um isomorfismo. Além disso, se o domínio $D(A)$ tem como norma $\|u\|_{D(A)}=\|A u\|_{H}$ (equivalente a norma do gráfico $\|u\|_{G}^{2}=\|u\|_{H}^{2}+\|A u\|_{H}^{2}$ ), então $D(A)$ é um espaço de Hilbert denso em $H$.

Observação 1.13. Um exemplo de uma forma bilinear satisfazendo as propriedades acima é dada pelo produto interno $(\cdot, \cdot)_{V}$ em $V$. Assim, o operador $A: D(A) \subset H \rightarrow H$ definido anteriormente é tal que

$$
(A u, v)_{H}=(u, v)_{V}, \quad \forall u \in D(A), \quad \forall v \in V .
$$

Como o operador $A$ satisfaz algumas propriedades e $V \hookrightarrow \hookrightarrow H$, então, da teoria espectral (ver por exemplo Yosida [49]), existe uma base ortonormal completa $\left\{w_{j}\right\}_{j \in \mathbb{N}}$ de $H$ e uma sequência de números reais $\left\{\lambda_{j}\right\}_{j \in \mathbb{N}}$ tais que

$$
\begin{aligned}
& 0<\lambda_{1} \leq \lambda_{2} \leq \cdots \quad \text { com } \quad \lambda_{j} \rightarrow \infty \quad \text { quando } j \rightarrow \infty, \\
& w_{j} \in D(A) \quad \text { e } \quad A w_{j}=\lambda_{j} w_{j}, \quad \forall j \in \mathbb{N}, \\
& \left(w_{i}, w_{j}\right)_{H}=\delta_{i j} \quad \text { e } \quad a\left(w_{i}, w_{j}\right)=\lambda_{i} \delta_{i j}, \quad \forall i, j \in \mathbb{N},
\end{aligned}
$$

onde $\delta_{i j}$ é o delta de Kronecker.

Considerando as hipóteses e a base $\left\{w_{j}\right\}_{j \in \mathbb{N}}$ mencionadas acima, podemos definir os operadores com potências fracionárias $A^{s}, s \in \mathbb{R}$, do operador $A$ e caracterizar os operadores $A^{s}$ em termos da base $\left\{w_{j}\right\}_{j \in \mathbb{N}}$.

Para $s>0$, o operador $A^{s}: D\left(A^{s}\right) \subset H \rightarrow H$ é um operador linear não limitado, positivo definido, autoadjunto e injetivo, cujo domínio $D\left(A^{s}\right)$ é denso em $H$ e, munido com o produto interno e norma

$$
(u, v)_{D\left(A^{s}\right)}=\left(A^{s} u, A^{s} v\right)_{H} \quad \text { e } \quad\|u\|_{D\left(A^{s}\right)}=\left\|A^{s} u\right\|_{H}
$$

$D\left(A^{s}\right)$ é um espaço de Hilbert. Além disso, $D\left(A^{-s}\right)$ é definido como o dual de $D\left(A^{s}\right)$ e o operador $A^{s}$ pode ser extendido como um isomorfismo de $H$ em $D\left(A^{-s}\right)$. $\operatorname{Em} D\left(A^{-s}\right)$, consideramos o produto interno e norma como acima substituindo $s$ por $-s$.

Usando que $V \hookrightarrow \hookrightarrow H$, podemos definir $A^{s}$, para $s>0$, em termos da base $\left\{w_{j}\right\}_{j \in \mathbb{N}}$ por

$$
A^{s} u=\sum_{j=1}^{\infty} \lambda_{j}^{s}\left(u, w_{j}\right)_{H} w_{j}, \quad \forall u \in D\left(A^{s}\right),
$$

onde

$$
D\left(A^{s}\right)=\left\{\left.u \in H\left|\sum_{j=1}^{\infty} \lambda_{j}^{2 s}\right|\left(u, w_{j}\right)_{H}\right|^{2}<\infty\right\} .
$$


Neste caso, a norma em $D\left(A^{s}\right)$ pode ser reescrita como

$$
\|u\|_{D\left(A^{s}\right)}=\left(\sum_{j=1}^{\infty} \lambda_{j}^{2 s}\left|\left(u, w_{j}\right)_{H}\right|^{2}\right)^{1 / 2}, \quad \forall u \in D\left(A^{s}\right) .
$$

Sejam $X, Y$ espaços de Hilbert tais que $X \hookrightarrow \hookrightarrow Y$ e

$$
\begin{gathered}
X:=V=D\left(A^{1 / 2}\right), Y:=H=D\left(A^{0}\right), \\
a(u, v)=((u, v))_{X} .
\end{gathered}
$$

Então, os espaços $D\left(A^{s}\right)$, para $s \in[0,1 / 2]$, são espaços intermediários entre $X$ e $Y$ chamados de espaços de interpolação. Escrevemos

$$
D\left(A^{(1-\theta) / 2}\right)=[X, Y]_{\theta}, \forall \theta \in[0,1],
$$

que é um espaço de Hilbert munido do produto interno

$$
((u, v))_{[X, Y]_{\theta}}=\left(\left(A^{(1-\theta) / 2} u, A^{(1-\theta) / 2} v\right)\right)_{Y} .
$$

e sua norma satisfaz

$$
\|u\|_{[X, Y]_{\theta}} \leq c(\theta)\|u\|_{X}^{1-\theta}\|u\|_{Y}^{\theta}, \forall u \in X, \forall \theta \in[0,1] .
$$

\subsection{Sistemas dinâmicos e atratores globais}

Nesta seção fazemos um apanhado das principais definições e resultados sobre sistemas dinâmicos definidos por um semigrupo fortemente contínuo de um espaço de Banach $X$. A apresentação da teoria pode ser encontrada em clássicos como Babin \& Vishik [5], Hale [20] e Temam [42]. Aqui, seguimos mais de perto os trabalhos recentes de Chueshov \& Lasiecka $[11,13]$.

Definição 1.14. Uma família de operadores não necessariamente lineares $\{S(t)\}_{t \geq 0}$, fortemente contínua em $X$, é chamada de $C_{0}$-semigrupo (não linear) se:

(i) $S(0)=I$ (operador identidade de $X$ );

(ii) $S(t+s)=S(t) S(s)$ para cada $t, s \geq 0$;

(iii) A aplicação $[0, \infty) \times X \ni(t, x) \mapsto S(t)(x) \in X$ é contínua para cada $x \in X$ fixado. O par $(X, S(t))$ também é chamado de sistema dinâmico, definido pelo semigrupo de evolução $S(t)$. 
Definição 1.15. Seja $(X, S(t))$ um sistema dinâmico. Dizemos que um subconjunto $\mathcal{A} \subset X$ é invariante (ou positivamente invariante ) pelo semigrupo $S(t)$, quando $S(t) \mathcal{A}=\mathcal{A}$ (ou $S(t) \mathcal{A} \subset \mathcal{A}$ ) para todo $t \geq 0$.

Definição 1.16. Seja $(X, S(t))$ um sistema dinâmico. Um conjunto fechado e limitado $\mathcal{A} \subset X$ é chamado de atrator global de $(X, S(t))$ quando:

(i) $\mathcal{A}$ é um conjunto invariante por $S(t)$;

(ii) $\mathcal{A}$ atrai (uniformemente) qualquer subconjunto limitado de $X$ sob a ação de $S(t)$, ou seja, para qualquer limitado $B \subset X$,

$$
\operatorname{dist}_{H}(S(t) B, \mathcal{A}):=\sup _{x \in S(t) B} \inf _{y \in \mathcal{A}} d(x, y) \rightarrow 0 \text { quando } t \rightarrow+\infty .
$$

onde $\operatorname{dist}_{H}(A, B)$ é chamada de semi-distância de Hausdorff entre os subconjuntos $A, B \subset X$.

Definição 1.17. Seja $(X, S(t))$ um sistema dinâmico. Um conjunto $\mathcal{B} \subset X$ é chamado de conjunto absorvente de $(X, S(t))$ se, para qualquer subconjunto limitado $B \subset X$, existe $T_{0}=T_{0}(B) \geq 0$ tal que

$$
S(t) B \subset \mathcal{B}, \quad \forall t \geq T_{0} .
$$

Quando um sistema dinâmico $(X, S(t))$ possui um conjunto absorvente limitado, dizemos que $(X, S(t))$ é um sistema dinâmico dissipativo.

Definição 1.18. Um sistema dinâmico $(X, S(t))$ é assintoticamente compacto se existe um conjunto atrator compacto $K$. Isto é, para cada conjunto limitado $B$ de $X$,

$$
\lim _{t \rightarrow \infty} d_{X}\{S(t) B, K\}=0
$$

onde $d_{X}\{A, D\}=\sup _{x \in A} \operatorname{dist}_{X}(x, D), \operatorname{com} A, D \subset X$.

O resultado seguinte é bem conhecido.

Teorema 1.19 ([35], Teorema 2.19). Um sistema dinâmico dissipativo $(X, S(t))$ possui um atrator global compacto $\mathcal{A} \subset X$ se, e somente se, é assintoticamente compacto. Além disso, temos a caracterização

$$
\mathcal{A}=\omega(\mathcal{B}),
$$

onde $\omega(\mathcal{B})$ denota o conjunto $\omega$-limite de $\mathcal{B}$, onde $\mathcal{B}$ é qualquer conjunto absorvente.

Em sistemas provenientes de equações hiperbólicas fracamente dissipativas, a verificação da compacidade assintótica pode ser bastante difícil. Nesse sentido, temos o conceito de regularidade assintótica (asymptotic smoothness). 
Definição 1.20. Um sistema dinâmico $(X, S(t))$ é assintoticamente regular se, para qualquer conjunto limitado e positivamente invariante $B \subset X$, existe um conjunto compacto $K \subset \bar{B}^{X}$ tal que

$$
\lim _{t \rightarrow \infty} d_{X}\{S(t) B, K\}=0 .
$$

Para sistemas dissipativos, como apontado em Chueshov \& Lasiecka [13, Prop. 7.1.4], os conceitos de compacidade assintótica e regularidade assintótica são equivalentes. O próximo resultado será usado neste trabalho.

Teorema 1.21 ([13], Teorema 7.1.11). Seja $(X, S(t))$ um sistema dinâmico. Suponhamos que para algum conjunto limitado positivamente invariante $B \subset X$ e para algum $\varepsilon>0$, exista $T=T(\varepsilon, B)$ tal que

$$
\left\|S(T) z^{1}-S(T) z^{2}\right\|_{X} \leq \varepsilon+\phi_{T}\left(z^{1}, z^{2}\right), \quad \forall z^{1}, z^{2} \in B
$$

onde $\phi_{T}: B \times B \rightarrow \mathbb{R}$ satisfaz

$$
\liminf _{n \rightarrow \infty} \liminf _{m \rightarrow \infty} \phi_{T}\left(z^{n}, z^{m}\right)=0
$$

para alguma sequência $\left(z^{n}\right)$ em $B$. Então, $(X, S(t))$ é um sistema dinâmico assintoticamente regular. Se, além disso, $(X, S(t))$ é dissipativo, então o sistema é também assintoticamente compacto.

\subsection{Dimensão fractal e atratores exponenciais}

O objetivo desta seção é apresentar resultados sobre dimensão fractal de atratores e sobre a existência de atratores exponenciais. Para tal, vamos trabalhar com a definição de quasi-estabilidade. As definições e resultados encontram-se em Chueshov \& Lasiecka ([13], Capítulo 7).

Definição 1.22. Seja $\mathcal{A}$ um subconjunto compacto de um espaço métrico $X$. A dimensão fractal de $\mathcal{A}$, denotada por $\operatorname{dim}_{f} \mathcal{A}$, é definida por

$$
\operatorname{dim}_{f} \mathcal{A}=\lim _{\varepsilon \rightarrow 0} \sup \frac{\ln n(\mathcal{A}, \varepsilon)}{\ln (1 / \varepsilon)},
$$

onde $n(\mathcal{A}, \varepsilon)$ é o número mínimo de bolas fechadas de raio $\varepsilon$ necessário para cobrir $\mathcal{A}$.

Definição 1.23. Uma seminorma $n_{X}(\cdot)$ definida sobre um espaço de Banach $X$ é compacta se $n_{X}\left(x_{j}\right) \rightarrow 0$ para qualquer sequência $x_{j} \rightarrow 0$ em $X$. 
No que segue, vamos definir o conceito de quasi-estabilidade. Sejam $X, Y, Z$ espaços de Banach reflexivos com $X \hookrightarrow \hookrightarrow Y$ e $H=X \times Y \times Z$. Consideremos o sistema dinâmico $(H, S(t))$ dado pelo operador de evolução

$$
S(t) z=\left(u(t), u_{t}(t), \xi^{t}\right), \quad z=\left(u_{0}, u_{1}, \xi_{0}\right) \in H
$$

e satisfazendo

$$
u \in C\left(\mathbb{R}^{+} ; X\right) \cap C^{1}\left(\mathbb{R}^{+} ; Y\right), \quad \xi \in C\left(\mathbb{R}^{+} ; Z\right) .
$$

Definição 1.24. Dizemos que o sistema dinâmico $(H, S(t))$ definido por (1.3) é quasiestável sobre um conjunto $B \subset H$ se existem uma seminorma compacta $n_{X}(\cdot)$ sobre $X$ e funções não negativas $a(t), b(t), c(t)$ sobre $\mathbb{R}^{+}$, onde $a(t), c(t)$ são localmente limitadas em $[0, \infty)$ e $b(t) \in L^{1}\left(\mathbb{R}^{+}\right)$com $\lim _{t \rightarrow \infty} b(t)=0$, tais que

$$
\left\|S(t) z^{1}-S(t) z^{2}\right\|_{H}^{2} \leq a(t)\left\|z^{1}-z^{2}\right\|_{H}^{2}
$$

e

$$
\left\|S(t) z^{1}-S(t) z^{2}\right\|_{H}^{2} \leq b(t)\left\|z^{1}-z^{2}\right\|_{H}^{2}+c(t) \sup _{0 \leq s \leq t}\left[n_{X}\left(u^{1}(s)-u^{2}(s)\right)\right]^{2}
$$

para $t>0$ e $z^{i} \in B$, onde $z^{i}=\left(u_{0}^{i}, u_{1}^{i}, \xi_{0}^{i}\right), i=1,2$.

Teorema 1.25 ([13], Teorema 7.9.6). Seja $(H, S(t))$ um sistema dinâmico dado por (1.3) $e$ satisfazendo a regularidade (1.4). Se $(H, S(t))$ possui um atrator global compacto $\mathcal{A}$ e é quasi-estável sobre $\mathcal{A}$, então o atrator $\mathcal{A}$ possui dimensão fractal finita.

Definição 1.26. Dizemos que um conjunto compacto $\mathcal{A}_{\text {exp }} \subset H$ é um atrator exponencial de um sistema dinâmico $(H, S(t))$ se $\mathcal{A}_{\text {exp }}$ é positivamente invariante, possui dimensão fractal finita, e para todo conjunto limitado $B \subset H$, existem constantes positivas $t_{B}, C_{B}, \sigma_{B}$ tais que

$$
d_{H}\left\{S(t) B, \mathcal{A}_{\exp }\right\} \equiv \sup _{z \in B} \operatorname{dist}_{H}\left(S(t) z, \mathcal{A}_{\exp }\right) \leq C_{B} e^{-\sigma_{B}\left(t-t_{B}\right)}, \quad t \geq t_{B}
$$

Indicamos os trabalhos de Eden et al [15] e Miranville \& Zelik [35] para uma abordagem mais completa. Em muitos caso, para problemas hiperbólicos fracamente dissipativos, é difícil verificar que $\mathcal{A}_{\text {exp }}$ possui dimensão fractal finita. Entretanto, a quasi-estabilidade oferece uma maneira simples de obtermos um atrator exponencial $\mathcal{A}_{\text {exp }}$ cuja dimensão é finita em um espaço (possivelmente) maior $\widetilde{H} \supseteq H$. Neste caso, dizemos que $\mathcal{A}_{\text {exp }}$ é um atrator exponencial generalizado.

Teorema 1.27 ([13], Teorema 7.9.9). Seja $(H, S(t))$ o sistema dinâmico definido por (1.3) e satisfazendo a regularidade (1.4). Suponhamos que $(H, S(t))$ é dissipativo, quasi-estável 
sobre algum conjunto absorvente $\mathcal{B}$ e que existe um espaço $\widetilde{H} \supseteq H$ tal que a aplicação $t \mapsto S(t) z$ é Hölder contínua em $\widetilde{H}$ para cada $z \in \mathcal{B}$, isto é, existem $0<\alpha \leq 1$ e $C_{\mathcal{B}, T}>0$ tais que

$$
\left\|S\left(t_{2}\right) z-S\left(t_{1}\right) z\right\|_{\widetilde{H}} \leq C_{\mathcal{B}, T}\left|t_{2}-t_{1}\right|^{\alpha}, \quad t_{1}, t_{2} \in[0, T], z \in \mathcal{B} .
$$

Então, o sistema dinâmico $(H, S(t))$ possui um atrator exponencial (generalizado) com dimensão finita no espaço $\widetilde{H}$.

\subsection{Uma caracterização do atrator global}

Nesta seção, apresentamos uma outra caracterização do atrator global por meio do conceito de variedade instável.

Definição 1.28. Seja $\mathcal{N}$ o conjunto dos pontos estacionários do sistema dinâmico $(X, S(t))$, isto é,

$$
\mathcal{N}=\{z \in X ; S(t) z=z, \quad \forall t \geq 0\} .
$$

A variedade instável $\mathbb{M}_{+}(\mathcal{N})$ emanando de $\mathcal{N}$ é um subconjunto de $X$ tal que, para cada $z \in \mathbb{M}_{+}(\mathcal{N})$, existe uma trajetória $\gamma=\{\mathbf{u}(t) \mid t \in \mathbb{R}\}$ satisfazendo

$$
\mathbf{u}(0)=z \quad \mathrm{e} \quad \lim _{t \rightarrow-\infty} \operatorname{dist}_{H}(\mathbf{u}(t), \mathcal{N})=0
$$

Definição 1.29. Dizemos que um sistema dinâmico $(X, S(t))$ é gradiente se existe um funcional de Lyapunov estrito sobre $X$, isto é, existe um funcional contínuo $\Psi(z)$ tal que:

(i) $t \mapsto \Psi(S(t) z)$ é não-crescente para algum $z \in X$;

(ii) Se $z \in X$ e $\Psi(S(t) z)=\Psi(z)$, para todo $t>0$, então $S(t) z=z$, para todo $t>0$.

A estrutura de um atrator global para um sistema gradiente é estabelecido no seguinte resultado.

Teorema 1.30 ([13], Teorema 7.5.6). Seja $\mathcal{N}$ o conjunto de pontos estacionários do sistema dinâmico $(X, S(t))$. Suponhamos que $(X, S(t))$ possui um atrator global compacto $\mathcal{A}$. Se existe um funcional de Lyapunov estrito sobre $\mathcal{A}$, então $\mathcal{A}=\mathbb{M}_{+}(\mathcal{N})$. 


$\frac{10}{2}$

\section{Um problema quasilinear}

\section{viscoelástico com história}

Neste capítulo, estudamos a boa colocação (no sentido de Hadamard) do problema misto correspondente à equação (1). Devido ao termo de memória, tal problema misto é não autônomo. Isso foi observado anteriormente nos trabalhos pioneiros de Giorgi et al [18, 19] e Pata \& Zucchi [38]. Para trabalhar num contexto de sistemas autônomos, seguindo um argumento de Dafermos [14], eles introduziram uma nova variável

$$
\eta=u(x, t)-u(x, t-s)
$$

ao problema. A formulação precisa do novo problema com variável $\eta$ é discutida na próxima seção. O nosso resultado principal é o Teorema 2.2. 


\subsection{O problema com história}

Adotamos o mesmo procedimento apresentado por Giorgi et al [19] e Pata \& Zucchi [38]. Seja $\Omega$ um domínio limitado de $\mathbb{R}^{N}, N \geq 1$, com fronteira regular $\Gamma$. Seja $\eta$ o deslocamento relativo da história do sistema, definido por

$$
\eta=\eta^{t}(x, s)=u(x, t)-u(x, t-s), \quad(x, s) \in \Omega \times \mathbb{R}^{+}, \quad t \geq 0 .
$$

Derivando formalmente em relação a $t$ e $s$ obtemos

$$
\eta_{t}^{t}(x, s)=-\eta_{s}^{t}(x, s)+u_{t}(x, t), \quad(x, s) \in \Omega \times \mathbb{R}^{+}, \quad t \geq 0 .
$$

Para essa equação definimos a condição inicial $(t=0)$

$$
\eta^{0}(x, s)=u_{0}(x, 0)-u_{0}(x,-s), \quad(x, s) \in \Omega \times \mathbb{R}^{+},
$$

onde $u_{0}(x, t)$ para $t \leq 0$ é prescrito.

Assim, o termo original da memória é reescrito como

$$
\begin{aligned}
\int_{-\infty}^{t} \mu(t-s) \Delta u(s) d s & =\int_{0}^{\infty} \mu(s) \Delta u(t-s) d s \\
& =\left(\int_{0}^{\infty} \mu(s) d s\right) \Delta u-\int_{0}^{\infty} \mu(s) \Delta \eta^{t}(s) d s
\end{aligned}
$$

e a equação (1)

$$
\left|u_{t}\right|^{\rho} u_{t t}-\alpha \Delta u-\Delta u_{t t}+\int_{-\infty}^{t} \mu(t-s) \Delta u(s) d s-\gamma \Delta u_{t}+f(u)=h,
$$

torna-se

$$
\left|u_{t}\right|^{\rho} u_{t t}-\left(\alpha-\int_{0}^{\infty} \mu(s) d s\right) \Delta u-\Delta u_{t t}-\int_{0}^{\infty} \mu(s) \Delta \eta^{t}(s) d s-\gamma \Delta u_{t}+f(u)=h .
$$

Por simplicidade, vamos considerar

$$
\alpha-\int_{0}^{\infty} \mu(s) d s=1
$$

Consequentemente, o sistema correspondente à equação (1) se escreve como

$$
\begin{aligned}
& \left|u_{t}\right|^{\rho} u_{t t}-\Delta u-\Delta u_{t t}-\int_{0}^{\infty} \mu(s) \Delta \eta(s) d s-\gamma \Delta u_{t}+f(u)=h, \text { em } \Omega \times \mathbb{R}^{+}, \\
& \eta_{t}=-\eta_{s}+u_{t}, \text { em } \Omega \times \mathbb{R}^{+} \times \mathbb{R}^{+},
\end{aligned}
$$


com condições de fronteira

$$
u=0 \quad \text { sobre } \Gamma \times \mathbb{R}^{+}, \quad \eta=0 \quad \text { sobre } \Gamma \times \mathbb{R}^{+} \times \mathbb{R}^{+}
$$

e dados iniciais

$$
u(x, 0)=u_{0}(x), \quad u_{t}(x, 0)=u_{1}(x), \quad \eta^{t}(x, 0)=0, \quad \eta^{0}(x, s)=\eta_{0}(x, s),
$$

onde

$$
\left\{\begin{array}{l}
u_{0}(x)=u_{0}(x, 0), \quad x \in \Omega, \\
u_{1}(x)=\left.\partial_{t} u_{0}(x, t)\right|_{t=0}, \quad x \in \Omega, \\
\eta_{0}(x, s)=u_{0}(x, 0)-u_{0}(x,-s), \quad(x, s) \in \Omega \times \mathbb{R}^{+} .
\end{array}\right.
$$

A seguir, vamos apresentar nossos resultados para o sistema (2.2)-(2.5), que é autônomo.

\subsection{Primeiro teorema: boa colocação e estabilidade}

Vamos apresentar as hipóteses do nosso primeiro resultado. Com relação ao termo $\left|u_{t}\right|^{\rho} u_{t t}$, assumimos que

$$
\rho>0 \text { se } N=1,2 \text { e } 1<\rho \leq \frac{2}{N-2} \text { se } N \geq 3 .
$$

Assim, vale a imersão

$$
H_{0}^{1}(\Omega) \hookrightarrow L^{2(\rho+1)}(\Omega) .
$$

A condição (2.6) é considerada em todos os artigos relacionados à equação (3). Com respeito a $f: \mathbb{R} \rightarrow \mathbb{R}$, assumimos que

$$
|f(u)-f(v)| \leq c_{0}\left(1+|u|^{p}+|v|^{p}\right)|u-v|, \quad \forall u, v \in \mathbb{R}
$$

onde $c_{0}>0$,

$$
0<p \leq \frac{2}{N-2} \text { se } N \geq 3 \text { e } p>0 \text { se } N=1,2 .
$$

Assumimos também que

$$
f(u) u \geq \hat{f}(u) \geq 0, \quad \forall u \in \mathbb{R},
$$

onde $\hat{f}(z)=\int_{0}^{z} f(s) d s$. As hipóteses (2.7) e (2.9) incluem termos não-lineares da forma

$$
f(u) \approx|u|^{p} u+|u|^{\alpha} u, \quad 0<\alpha<p .
$$

Agora, com respeito ao termo de memória, assumimos que

$$
\mu \in C^{1}\left(\mathbb{R}^{+}\right) \cap L^{1}\left(\mathbb{R}^{+}\right), \quad 0<\mu(s)<\infty,
$$


e que existem $k_{0}, k_{1}>0$ tais que

$$
\int_{0}^{\infty} \mu(s) d s=k_{0}
$$

$\mathrm{e}$

$$
\mu^{\prime}(s) \leq-k_{1} \mu(s), \quad \forall s \in \mathbb{R}^{+}
$$

Neste momento, precisamos definir um espaço com peso para a variável $\eta$. Seja

$$
\mathcal{M}=L_{\mu}^{2}\left(\mathbb{R}^{+} ; H_{0}^{1}(\Omega)\right)=\left\{\xi: \mathbb{R}^{+} \rightarrow H_{0}^{1}(\Omega) \mid \int_{0}^{\infty} \mu(s)\|\nabla \xi(s)\|_{2}^{2} d s<\infty\right\} .
$$

Este espaço é de Hilbert quando munido de produto interno e norma

$$
(\xi, \zeta)_{\mathcal{M}}=\int_{0}^{\infty} \mu(s)\left(\int_{\Omega} \nabla \xi(s) \nabla \zeta(s) d x\right) d s \quad \text { e } \quad\|\xi\|_{\mathcal{M}}^{2}=\int_{0}^{\infty} \mu(s)\|\nabla \xi(s)\|_{2}^{2} d s
$$

respectivamente. Consideremos o espaço de fase (dos dados iniciais)

$$
\mathcal{H}=H_{0}^{1}(\Omega) \times H_{0}^{1}(\Omega) \times \mathcal{M}
$$

o qual é natural com respeito as soluções fracas.

Definição 2.1. Com os dados iniciais $\left(u_{0}, u_{1}, \eta_{0}\right) \in \mathcal{H}$ e $h \in L^{2}(\Omega)$, uma função $z=$ $\left(u, u_{t}, \eta\right) \in C([0, T], \mathcal{H})$ é uma solução fraca de (2.2)-(2.5) se satisfaz a condição inicial $z(0)=\left(u_{0}, u_{1}, \eta_{0}\right) \mathrm{e}$

$$
\begin{aligned}
& \left(\left|u_{t}(t)\right|^{\rho} u_{t t}(t), \omega\right)+(\nabla u(t), \nabla \omega)+\left(\nabla u_{t t}(t), \nabla \omega\right) \\
& \quad+\int_{0}^{\infty} \mu(s)\left(\nabla \eta^{t}(s), \nabla \omega\right) d s+(f(u(t))-h, \omega)=0 \\
& \left(\partial_{t} \eta^{t}+\partial_{s} \eta^{t}, \xi\right)_{\mathcal{M}}=\left(u_{t}(t), \xi\right)_{\mathcal{M}},
\end{aligned}
$$

para todo $\omega \in H_{0}^{1}(\Omega), \xi \in \mathcal{M}$, e $t \in[0, T]$ quase sempre.

A energia do sistema é definida por

$$
\begin{aligned}
E(t)= & \frac{1}{\rho+2}\left\|u_{t}(t)\right\|_{\rho+2}^{\rho+2}+\frac{1}{2}\|\nabla u(t)\|_{2}^{2}+\frac{1}{2}\left\|\nabla u_{t}(t)\right\|_{2}^{2}+\frac{1}{2}\left\|\eta^{t}\right\|_{\mathcal{M}}^{2} \\
& +\int_{\Omega} \hat{f}(u(t)) d x-\int_{\Omega} h u(t) d x .
\end{aligned}
$$


Teorema 2.2. Suponhamos que as hipóteses (2.6)-(2.12) sejam válidas e $\gamma \geq 0$. Temos:

(i) Se os dados iniciais $\left(u_{0}, u_{1}, \eta_{0}\right) \in \mathcal{H}$ e $h \in L^{2}(\Omega)$, então o problema (2.2)-(2.5) tem uma solução fraca

$$
\left(u, u_{t}, \eta\right) \in C([0, T], \mathcal{H}), \quad \forall T>0
$$

satisfazendo

$$
\begin{aligned}
& u \in L^{\infty}\left(\mathbb{R}^{+} ; H_{0}^{1}(\Omega)\right), u_{t} \in L^{\infty}\left(\mathbb{R}^{+} ; H_{0}^{1}(\Omega)\right), \\
& u_{t t} \in L^{2}\left([0, T] ; H_{0}^{1}(\Omega)\right), \eta \in L^{\infty}\left(\mathbb{R}^{+} ; \mathcal{M}\right) .
\end{aligned}
$$

(ii) Se $\rho>1$, então as soluções fracas dependem continuamente dos dados iniciais em $\mathcal{H}$. Em particular, o problema (2.2)-(2.5) tem unicidade.

(iii) Se $h=0$, então a energia do sistema satisfaz

$$
E(t) \leq K E(0) e^{-\nu t}, \quad t \geq 0,
$$

onde $K, \nu>0$ dependem de $E(0)$.

Observação 2.3. (i) Admitimos a hipótese $\rho>1$ para obtermos a unicidade de soluções. Isto torna $\left|u_{t}\right|^{\rho}$ localmente Lipschitz, que é essencial em nossos argumentos.

(ii) Na demonstração do decaimento exponencial da energia, não pedimos $\rho>1$ ou $\gamma>0$. Logo, o decaimento é provado para a solução obtida no item $(i)$. Notamos que a taxa de decaimento só é uniforme em conjuntos limitados de $\mathcal{H}$.

\subsection{Prova do teorema: boa colocação}

Aplicamos aqui o método de Faedo-Galerkin, que em linhas gerais, é descrito em Lions [26] por meio de vários exemplos. Combinamos os argumentos desenvolvidos em Cavalcanti et al [8], Han \& Wang [22] e Pata \& Zucchi [38].

\subsubsection{Existência global}

Seja $\left\{\omega_{j}\right\}_{j=1}^{\infty}$ a base de auto-funções de $-\Delta u=\lambda u$ em $\Omega$, com condições de fronteira de Dirichlet. Então, sabemos que essa base é ortonormal em $L^{2}(\Omega)$ e ortogonal em $H_{0}^{1}(\Omega)$. Além disso,

$$
\left\{\begin{array}{cc}
-\Delta \omega_{j}=\lambda_{j} \omega_{j} & , \quad x \in \Omega \\
\omega_{j}=0 & , \quad x \in \Gamma
\end{array},\right.
$$


onde $\lambda_{j} \geq 0, \forall j \in \mathbb{N}$ e $\lambda_{i} \neq \lambda_{j}$ para $i \neq j$.

Agora, vamos construir uma base ortonormal $\left\{\xi_{j}\right\}_{j=1}^{\infty}$ para $\mathcal{M}=L_{\mu}^{2}\left(\mathbb{R}^{+} ; H_{0}^{1}(\Omega)\right)$. Para tal, usaremos um raciocínio semelhante ao de Pata \& Zucchi [38]. Sejam $\left\{h_{j}\right\}_{j=1}^{\infty}$ uma base ortonormal de $L_{\mu}^{2}\left(\mathbb{R}^{+}\right) \cap C_{0}^{\infty}\left(\mathbb{R}^{+}\right)$e $\left\{\hat{\omega}_{j}\right\}_{j=1}^{\infty}$ tal que $\hat{\omega}_{j}=\frac{\omega_{j}}{\left\|\nabla \omega_{j}\right\|_{2}}, \forall j \in \mathbb{N}$. Para $\xi_{i}=h_{p} \hat{\omega}_{k}$ e $\xi_{j}=h_{q} \hat{\omega}_{l}$, obtemos:

$$
\begin{aligned}
\left(\xi_{i}, \xi_{j}\right)_{\mathcal{M}} & =\int_{0}^{\infty} \mu(s)\left(\int_{\Omega} \nabla \xi_{i} \nabla \xi_{j} d x\right) d s \\
& =\int_{0}^{\infty} \mu(s) h_{p}(s) h_{q}(s)\left(\int_{\Omega} \nabla \hat{\omega}_{k} \nabla \hat{\omega}_{l} d x\right) d s \\
& =\delta_{p q} \delta_{k l} \\
& =\delta_{i j} .
\end{aligned}
$$

Logo, $\left\{\xi_{j}\right\}_{j=1}^{\infty}$ é uma base ortonormal de $\mathcal{M}$ e $\xi_{j} \in C_{0}^{\infty}\left(\mathbb{R}^{+} ; H_{0}^{1}(\Omega)\right), \forall j \in \mathbb{N}$.

\section{Problema Aproximado:}

Para cada $m \in \mathbb{N}$, consideremos os subespaços

$$
\operatorname{Span}\left\{\omega_{1}, \ldots, \omega_{m}\right\} \subset H_{0}^{1}(\Omega) \quad \text { e } \quad \operatorname{Span}\left\{\xi_{1}, \ldots, \xi_{m}\right\} \subset \mathcal{M}
$$

Para os dados iniciais $\left(u_{0}, u_{1}, \eta_{0}\right) \in \mathcal{H}$, procuramos por soluções aproximadas

$$
u^{m}(t)=\sum_{j=1}^{m} y_{m j}(t) \omega_{j} \quad \text { e } \quad \eta^{t, m}(s)=\sum_{j=1}^{m} g_{m j}(t) \xi_{j}(s)
$$

tais que

$$
\begin{aligned}
& \left(\left|u_{t}^{m}(t)\right|^{\rho} u_{t t}^{m}(t), \omega_{j}\right)-\left(\Delta u^{m}(t), \omega_{j}\right)-\left(\Delta u_{t t}^{m}(t), \omega_{j}\right) \\
& \quad+\left(\eta^{t, m}(t), \omega_{j}\right)_{\mathcal{M}}-\left(\gamma \Delta u_{t}^{m}(t), \omega_{j}\right)+\left(f\left(u^{m}(t)\right), \omega_{j}\right)=\left(h, \omega_{j}\right) \\
& \left(\eta_{t}^{t, m}, \xi_{j}\right)_{\mathcal{M}}=-\left(\eta_{s}^{t, m}, \xi_{j}\right)_{\mathcal{M}}+\left(u_{t}^{m}(t), \xi_{j}\right)_{\mathcal{M}}
\end{aligned}
$$

para $1 \leq j \leq m$, com condições iniciais

$$
u^{m}(0)=u_{0}^{m}, \quad u_{t}^{m}(0)=u_{1}^{m}, \quad \eta^{0, m}=\eta_{0}^{m},
$$

onde $u_{0}^{m}, u_{1}^{m}, \eta_{0}^{m}$ são escolhidos de forma que

$$
u_{0}^{m} \rightarrow u_{0} \text { em } H_{0}^{1}(\Omega), \quad u_{1}^{m} \rightarrow u_{1} \text { em } H_{0}^{1}(\Omega), \quad \eta_{0}^{0, m} \rightarrow \eta_{0} \text { em } \mathcal{M} .
$$

Assim, o sistema (2.17)-(2.18) com condições inciais (2.19) é um sistema de equações diferencias ordinárias em $t$. Tal sistema possui existência local via Teorema de Caratheorody, 
como mostrado com detalhes em Han \& Wang [22]. A seguir, estimativas a priori mostrarão que as soluções locais $\left(u^{m}(t), \eta^{t, m}\right)$ podem ser extendidas ao intervalo $[0, \infty)$.

Primeira Estimativa: Multiplicando a equação (2.17) por $y_{m j}^{\prime}(t)$ e a equação (2.18) por $g_{m j}(s)$ e somando em relação a $j$ de 1 até $m$, obtemos

$$
\begin{gathered}
\left(\left|u_{t}^{m}(t)\right|^{\rho} u_{t t}^{m}(t), u_{t}^{m}(t)\right)-\left(\Delta u^{m}(t), u_{t}^{m}(t)\right)-\left(\Delta u_{t t}^{m}(t), u_{t}^{m}(t)\right)+\left(\eta^{t, m}(t), u_{t}^{m}(t)\right)_{\mathcal{M}} \\
-\left(\gamma \Delta u_{t}^{m}(t), u_{t}^{m}(t)\right)+\left(f\left(u^{m}(t)\right), u_{t}^{m}(t)\right)=\left(h, u_{t}^{m}(t)\right) \\
\left(\eta_{t}^{t, m}, \eta^{t, m}\right)_{\mathcal{M}}=-\left(\eta_{s}^{t, m}, \eta^{t, m}\right)_{\mathcal{M}}+\left(u_{t}^{m}(t), \eta^{t, m}\right)_{\mathcal{M}}
\end{gathered}
$$

Definimos a energia aproximada por

$$
\begin{aligned}
E^{m}(t)= & \frac{1}{\rho+2}\left\|u_{t}^{m}(t)\right\|_{\rho+2}^{\rho+2}+\frac{1}{2}\left\|\nabla u^{m}(t)\right\|_{2}^{2}+\frac{1}{2}\left\|\nabla u_{t}^{m}(t)\right\|_{2}^{2}+\frac{1}{2}\left\|\eta^{t, m}\right\|_{\mathcal{M}}^{2} \\
& +\int_{\Omega} \hat{f}\left(u^{m}(t)\right) d x-\int_{\Omega} h u^{m}(t) d x .
\end{aligned}
$$

Então, somando as duas equações acima e observando que

$$
\begin{aligned}
\frac{1}{\rho+2} \frac{d}{d t}\left\|u_{t}^{m}(t)\right\|_{\rho+2}^{\rho+2} & =\left(\left|u_{t}^{m}(t)\right|^{\rho} u_{t t}^{m}(t), u_{t}^{m}(t)\right), \\
\frac{1}{2} \frac{d}{d t}\left\|\nabla u^{m}(t)\right\|_{2}^{2} & =-\left(\Delta u^{m}(t), u_{t}^{m}(t)\right), \\
\frac{1}{2} \frac{d}{d t}\left\|\nabla u_{t}^{m}(t)\right\|_{2}^{2} & =-\left(\Delta u_{t t}^{m}(t), u_{t}^{m}(t)\right), \\
\frac{1}{2} \frac{d}{d t}\left\|\eta^{t, m}\right\|_{\mathcal{M}}^{2} & =\left(\eta_{t}^{t, m}, \eta^{t, m}\right)_{\mathcal{M}}, \\
\frac{d}{d t} \int_{\Omega} \hat{f}\left(u^{m}(t)\right) d x & =\left(f\left(u^{m}(t)\right), u_{t}^{m}(t)\right), \\
\frac{d}{d t} \int_{\Omega} h u^{m}(t) d x & =\left(h, u_{t}^{m}(t)\right),
\end{aligned}
$$

obtemos:

$$
\frac{d}{d t} E^{m}(t)+\gamma\left\|\nabla u_{t}^{m}(t)\right\|_{2}^{2}=-\left(\eta_{s}^{t, m}, \eta^{t, m}\right)_{\mathcal{M}}
$$

Por outro lado, como $\mu \in L^{1}\left(\mathbb{R}^{+}\right), \eta^{t, m}(0)=0$ e $\mu^{\prime} \leq 0$, então

$$
\begin{aligned}
\left(\eta_{s}^{t, m}, \eta^{t, m}\right)_{\mathcal{M}}= & \frac{1}{2} \int_{\Omega}\left(\int_{0}^{\infty} \mu(s) \frac{d}{d s}\left|\nabla \eta^{t, m}(s)\right|^{2} d s\right) d x \\
= & -\frac{1}{2} \int_{\Omega}\left(\int_{0}^{\infty} \mu^{\prime}(s)\left|\nabla \eta^{t, m}(s)\right|^{2} d s\right) d x \\
& +\frac{1}{2} \int_{\Omega}\left(\int_{0}^{\infty} \frac{d}{d s}\left(\mu(s)\left|\nabla \eta^{t, m}(s)\right|^{2}\right) d s\right) d x \\
= & -\frac{1}{2} \int_{\Omega}\left(\int_{0}^{\infty} \mu^{\prime}(s)\left|\nabla \eta^{t, m}(s)\right|^{2} d s\right) d x \geq 0 .
\end{aligned}
$$


Logo,

$$
\frac{d}{d t} E^{m}(t)+\gamma\left\|\nabla u_{t}^{m}(t)\right\|_{2}^{2}=\frac{1}{2} \int_{\Omega}\left(\int_{0}^{\infty} \mu^{\prime}(s)\left|\nabla \eta^{t, m}(s)\right|^{2} d s\right) d x \leq 0 .
$$

Integrando de 0 a $t$ a desigualdade acima obtemos

$$
E^{m}(t)+\gamma \int_{0}^{t}\left\|\nabla u_{t}^{m}(s)\right\|_{2}^{2} d s \leq E^{m}(0)
$$

ou equivalentemente,

$$
\begin{aligned}
& \frac{1}{\rho+2}\left\|u_{t}^{m}(t)\right\|_{\rho+2}^{\rho+2}+\frac{1}{2}\left\|\nabla u^{m}(t)\right\|_{2}^{2}+\frac{1}{2}\left\|\nabla u_{t}^{m}(t)\right\|_{2}^{2}+\frac{1}{2}\left\|\eta^{t, m}\right\|_{\mathcal{M}}^{2} \\
& +\int_{\Omega} \hat{f}\left(u^{m}(t)\right) d x+\gamma \int_{0}^{t}\left\|\nabla u_{t}^{m}(s)\right\|_{2}^{2} d s \leq E^{m}(0)+\int_{\Omega} h u^{m}(t) d x .
\end{aligned}
$$

Usando as hipóteses sobre $\hat{f}$ e $f$ e as desigualdade de Hölder e Young temos

$$
E^{m}(0) \leq C\left(\left\|u_{1}^{m}\right\|_{\rho+2}^{\rho+2}+\left\|\nabla u_{0}^{m}\right\|_{2}^{2}+\left\|\nabla u_{1}^{m}\right\|_{2}^{2}+\left\|\eta_{0}^{m}\right\|_{\mathcal{M}}^{2}+\left\|u_{0}^{m}\right\|_{p+1}^{p+1}+\|h\|_{2}^{2}\right),
$$

que por sua vez é limitada pelos dados $\left(u_{0}, u_{1}, \eta^{0}, h\right)$ devido a (2.20).

Notemos também que

$$
\int_{\Omega} \hat{f}\left(u^{m}(t)\right) d x \geq 0
$$

e

$$
\int_{\Omega} h u^{m}(t) d x \leq \frac{1}{\lambda_{1}}\|h\|_{2}^{2}+\frac{1}{4}\left\|\nabla u^{m}(t)\right\|_{2}^{2}
$$

Logo,

$$
\begin{gathered}
\frac{1}{\rho+2}\left\|u_{t}^{m}(t)\right\|_{\rho+2}^{\rho+2}+\frac{1}{2}\left\|\nabla u^{m}(t)\right\|_{2}^{2}+\frac{1}{2}\left\|\nabla u_{t}^{m}(t)\right\|_{2}^{2}+\frac{1}{2}\left\|\eta^{t, m}\right\|_{\mathcal{M}}^{2}+\gamma \int_{0}^{t}\left\|\nabla u_{t}^{m}(s)\right\|_{2}^{2} d s \\
\leq E^{m}(0)+\frac{1}{\lambda_{1}}\|h\|_{2}^{2}+\frac{1}{4}\left\|\nabla u^{m}(t)\right\|_{2}^{2} .
\end{gathered}
$$

Por (2.20), obtemos

$$
\begin{aligned}
\frac{1}{\rho+2}\left\|u_{t}^{m}(t)\right\|_{\rho+2}^{\rho+2}+\frac{1}{4}\left\|\nabla u^{m}(t)\right\|_{2}^{2}+\frac{1}{2}\left\|\nabla u_{t}^{m}(t)\right\|_{2}^{2} \\
+\frac{1}{2}\left\|\eta^{t, m}\right\|_{\mathcal{M}}^{2}+\gamma \int_{0}^{t}\left\|\nabla u_{t}^{m}(s)\right\|_{2}^{2} d s \leq C,
\end{aligned}
$$

onde

$$
C=C\left(\left\|u_{1}\right\|_{\rho+2},\left\|\nabla u_{0}\right\|_{2},\left\|\nabla u_{1}\right\|_{2},\left\|\eta_{0}\right\|_{\mathcal{M}},\|h\|_{2}\right) .
$$

Assim,

$$
\left\|u_{t}^{m}(t)\right\|_{\rho+2}^{\rho+2}+\left\|\nabla u^{m}(t)\right\|_{2}^{2}+\left\|\nabla u_{t}^{m}(t)\right\|_{2}^{2}+\left\|\eta^{t, m}\right\|_{\mathcal{M}}^{2} \leq D_{1}, \quad t \geq 0
$$


onde $D_{1}$ depende apenas dos dados iniciais e independe de $t$ e $m$. Com a estimativa acima concluímos que

$$
\begin{aligned}
& u^{m}(t) \text { é limitado em } L^{\infty}\left(\mathbb{R}^{+} ; H_{0}^{1}(\Omega)\right), \\
& u_{t}^{m}(t) \text { é limitado em } L^{\infty}\left(\mathbb{R}^{+}, H_{0}^{1}(\Omega)\right), \\
& \eta^{t, m}(s) \text { é limitado em } L^{\infty}\left(\mathbb{R}^{+} ; \mathcal{M}\right) .
\end{aligned}
$$

Segunda Estimativa: Multiplicando a equação (2.17) por $y_{m j}^{\prime \prime}(t)$ e somando em relação a $j$ de 1 até $m$, obtemos

$$
\begin{aligned}
\left(\left|u_{t}^{m}(t)\right|^{\rho},\left|u_{t t}^{m}(t)\right|^{2}\right) & +\left(\nabla u^{m}(t), \nabla u_{t t}^{m}(t)\right)+\left\|\nabla u_{t t}^{m}(t)\right\|_{2}^{2}+\left(\eta^{t, m}(s), u_{t t}^{m}(t)\right)_{\mathcal{M}} \\
& +\gamma\left(\nabla u_{t}^{m}(t), \nabla u_{t t}^{m}(t)\right)+\left(f\left(u^{m}(t)\right), u_{t t}^{m}(t)\right)=\left(h, u_{t t}^{m}(t)\right),
\end{aligned}
$$

ou seja,

$$
\begin{array}{r}
\left(\left|u_{t}^{m}(t)\right|^{\rho},\left|u_{t t}^{m}(t)\right|^{2}\right)+\left\|\nabla u_{t t}^{m}(t)\right\|_{2}^{2}+\frac{\gamma d}{2 d t}\left\|u_{t}^{m}(t)\right\|_{2}^{2}=\left(h, u_{t t}^{m}(t)\right)-\left(f\left(u^{m}(t)\right), u_{t t}^{m}(t)\right) \\
-\left(\nabla u^{m}(t), \nabla u_{t t}^{m}(t)\right)-\left(\eta^{t, m}(s), u_{t t}^{m}(t)\right)_{\mathcal{M}} .
\end{array}
$$

Note que, usando as desigualdades de Hölder e Young, e tomando $C_{s}$ como constantes de imersão de Sobolev,

$$
\begin{aligned}
\left(\eta^{t, m}(s), u_{t t}^{m}(t)\right)_{\mathcal{M}} & \leq \int_{0}^{\infty} \mu(s)\left\|\nabla \eta^{t, m}(s)\right\|_{2}\left\|\nabla u_{t t}^{m}(t)\right\|_{2} d s \\
& \leq \int_{0}^{\infty} \mu(s)\left(\epsilon_{0}\left\|\nabla \eta^{t, m}(s)\right\|_{2}^{2}+\frac{1}{4 \epsilon_{0}}\left\|\nabla u_{t t}^{m}(t)\right\|_{2}^{2}\right) d s \\
& =\epsilon_{0}\left\|\eta^{t, m}(s)\right\|_{\mathcal{M}}^{2}+\frac{k_{0}}{4 \epsilon_{0}}\left\|\nabla u_{t t}^{m}(t)\right\|_{2}^{2} \\
\left(\nabla u^{m}(t), \nabla u_{t t}^{m}(t)\right) & \leq\left\|\nabla u^{m}(t)\right\|_{2}^{2}\left\|\nabla u_{t t}^{m}(t)\right\|_{2}^{2} \\
& \leq \epsilon_{1}\left\|\nabla u^{m}(t)\right\|_{2}^{2}+\frac{1}{4 \epsilon_{1}}\left\|\nabla u_{t t}^{m}(t)\right\|_{2}^{2} \\
\left(h, u_{t t}^{m}(t)\right) & \leq \epsilon_{2}\|h\|_{2}^{2}+\frac{1}{4 \epsilon_{2}}\left\|u_{t t}^{m}(t)\right\|_{2}^{2} \\
& \leq \epsilon_{2}\|h\|_{2}^{2}+\frac{C_{s}}{4 \epsilon_{2}}\left\|\nabla u_{t t}^{m}(t)\right\|_{2}^{2} \\
\left(f\left(u^{m}(t)\right), u_{t t}^{m}(t)\right) & \leq \epsilon_{3}\left\|f\left(u^{m}(t)\right)\right\|_{2}^{2}+\frac{1}{4 \epsilon_{3}}\left\|u_{t t}^{m}(t)\right\|_{2}^{2} \\
& \leq \epsilon_{3}\left\|c_{0}\left|u^{m}(t)\right|^{p+1}\right\|_{2}^{2}+\frac{1}{4 \epsilon_{3}}\left\|u_{t t}^{m}(t)\right\|_{2}^{2} \\
& \leq \epsilon_{3}\left(c_{0}\right)^{2}\left\|u^{m}(t)\right\|_{2(p+1)}^{2(p+1)}+\frac{C_{s}}{4 \epsilon_{3}}\left\|\nabla u_{t t}^{m}(t)\right\|_{2}^{2} \\
& \leq \epsilon_{3}\left(c_{0}\right)^{2}\left(C_{s}\right)^{2(p+1)}\left\|\nabla u^{m}(t)\right\|_{2}^{2(p+1)}+\frac{C_{s}}{4 \epsilon_{3}}\left\|\nabla u_{t t}^{m}(t)\right\|_{2}^{2}
\end{aligned}
$$


Substituindo e colocando $\epsilon_{0}=2 k_{0}, \epsilon_{1}=2, \epsilon_{2}=\epsilon_{3}=2 C_{s}$

$$
\begin{gathered}
\left(\left|u_{t}^{m}(t)\right|^{\rho},\left|u_{t t}^{m}(t)\right|^{2}\right)+\frac{1}{2}\left\|\nabla u_{t t}^{m}(t)\right\|_{2}^{2}+\frac{\gamma d}{2 d t}\left\|u_{t}^{m}(t)\right\|_{2}^{2} \\
\leq 2 C_{s}\|h\|_{2}^{2}+2 C_{s}\left(c_{0}\right)^{2}\left(C_{s}\right)^{2(p+1)}\left\|\nabla u^{m}(t)\right\|_{2}^{2(p+1)} \\
\quad+2\left\|\nabla u^{m}(t)\right\|_{2}^{2}+2 k_{0}\left\|\eta^{t, m}(s)\right\|_{\mathcal{M}}^{2} \\
\leq \beta D_{1}^{p+1}
\end{gathered}
$$

Agora, integrando de 0 a $t$ temos

$$
\int_{0}^{t}\left(\left|u_{t}^{m}(s)\right|^{\rho},\left|u_{t t}^{m}(s)\right|^{2}\right) d s+\frac{1}{2} \int_{0}^{t}\left\|\nabla u_{t t}^{m}(s)\right\|_{2}^{2} d s+\frac{\gamma}{2} \int_{0}^{t} \frac{d}{d s}\left\|u_{t}^{m}(s)\right\|_{2}^{2} d s \leq \beta D_{1}^{p+1} \int_{0}^{t} d s
$$

e, assim,

$$
\begin{aligned}
\int_{0}^{t}\left\|\nabla u_{t t}^{m}(s)\right\|_{2}^{2} d s+\left\|u_{t}^{m}(s)\right\|_{2}^{2} & \leq \frac{2}{\min (1, \gamma)}\left(\beta D_{1}^{p+1} t+\left\|u_{1}^{m}\right\|_{2}^{2}\right), \\
\int_{0}^{t}\left\|\nabla u_{t t}^{m}(s)\right\|_{2}^{2} d s & \leq D_{2}, \quad t \in[0, T]
\end{aligned}
$$

onde $D_{2}$ depende de $T$ e dos dados iniciais, mas não de $m$. Portanto,

$$
\left(u_{t t}^{m}\right) \text { é limitada em } L^{2}\left(0, T ; H_{0}^{1}(\Omega)\right) \text {. }
$$

Terceira Estimativa: Observando o termo não linear temos

$$
\begin{aligned}
\left\|\left|u_{t}^{m}(t)\right|^{\rho} u_{t}^{m}(t)\right\|_{2}^{2} & =\left\|u_{t}^{m}(t)\right\|_{2(\rho+1)}^{2(\rho+1)} \\
& \leq C_{s}\left\|\nabla u_{t}^{m}(t)\right\|_{2}^{2(\rho+1)} \\
& \leq C_{s} D_{1}^{\rho+1}, \quad t \geq 0
\end{aligned}
$$

Então,

$$
\left(\left|u_{t}^{m}(t)\right|^{\rho} u_{t}^{m}(t)\right) \text { é limitada em } L^{\infty}\left(0, T ; L^{2}(\Omega)\right) .
$$

Conclusão: Com as estimativas a priori obtidas acima, podemos passar ao limite as soluções aproximadas e garantir a existência de uma solução global fraca satisfazendo os dados iniciais. Detalhes podem ser encontrados em Cavalcanti et al [8] e Han \& Wang [22].

Portanto, o item $(i)$ do Teorema 2.2 está provado. 


\subsubsection{Dependência contínua dos dados}

Nesta seção, provamos uma das nossas principais contribuições.

Sejam $(u, \eta)$ e $(v, \xi)$ duas soluções fracas de (2.2)-(2.5) com respeito aos dados iniciais $\left\{u_{0}, u_{1}, \eta_{0}, h_{1}\right\}$ e $\left\{v_{0}, v_{1}, \xi_{0}, h_{2}\right\}$ respectivamente. Sejam $w=u-v$ e $\zeta=\eta-\xi$. Então, $(w, \zeta)$ é uma solução fraca de

$$
\begin{aligned}
\left|u_{t}\right|^{\rho} w_{t t}- & \Delta w-\Delta w_{t t}-\int_{0}^{\infty} \mu(s) \Delta \zeta(s) d s \\
& \quad-\gamma \Delta w_{t}+\left(\left|u_{t}\right|^{\rho}-\left|v_{t}\right|^{\rho}\right) v_{t t}+f(u)-f(v)=h_{1}-h_{2}, \\
\zeta_{t}=-\zeta_{s} & +w_{t},
\end{aligned}
$$

com a condição de fronteira de Dirichlet e a condições iniciais

$$
w(0)=u_{0}-v_{0}, \quad w_{t}(0)=u_{1}-v_{1}, \quad \zeta^{0}=\eta_{0}-\xi_{0} .
$$

Para este problema definimos, o funcional energia

$$
F(t)=\frac{1}{2} \int_{\Omega}\left|u_{t}(t)\right|^{\rho}\left|w_{t}(t)\right|^{2} d x+\frac{1}{2}\|\nabla w(t)\|_{2}^{2}+\frac{1}{2}\left\|\nabla w_{t}(t)\right\|_{2}^{2}+\frac{1}{2}\left\|\zeta^{t}\right\|_{\mathcal{M}}^{2} .
$$

Para simplificar as notações, consideremos que a norma dos dados iniciais são limitadas para algum $R>0$. Então, dado $T>0$ usamos $C_{R T}$ para denotar as constantes positivas que dependem de $R$ e $T$. Assim, o funcional $F(t)$ é de fato equivalente a norma de $\mathcal{H}$, pois como

$$
\int_{\Omega}\left|u_{t}\right|^{\rho}\left|w_{t}\right|^{2} d x \leq\left\|u_{t}\right\|_{\rho+2}^{\rho}\left\|w_{t}\right\|_{\rho+2}^{2} \leq C_{R T}\left\|\nabla w_{t}(t)\right\|_{2}^{2}
$$

então

$$
\frac{1}{2}\left\|\left(w(t), w_{t}(t), \zeta^{t}\right)\right\|_{\mathcal{H}}^{2} \leq F(t) \leq \min \left\{\frac{1}{2}, C_{R T}\right\}\left\|\left(w(t), w_{t}(t), \zeta^{t}\right)\right\|_{\mathcal{H}}^{2}
$$

Agora, vamos multiplicar a primeira equação por $w_{t}$ e a segunda por $\zeta$. Logo, concluímos que

$$
\begin{gathered}
\int_{\Omega}\left|u_{t}\right|^{\rho} w_{t t} w_{t} d x+\frac{1}{2} \frac{d}{d t}\|\nabla w\|_{2}^{2}+\frac{1}{2} \frac{d}{d t}\left\|\nabla w_{t}\right\|_{2}^{2}+\frac{1}{2} \frac{d}{d t}\left\|\zeta^{t}\right\|_{\mathcal{M}}^{2} \\
=-\int_{\Omega}\left(\left|u_{t}\right|^{\rho}-\left|v_{t}\right|^{\rho}\right) v_{t t} w_{t} d x-\int_{\Omega}(f(u)-f(v)) w_{t} d x \\
-\gamma\left\|\nabla w_{t}\right\|_{2}^{2}-\left(\zeta^{t}, \zeta_{s}^{t}\right)_{\mathcal{M}}+\int_{\Omega}\left(h_{1}-h_{2}\right) w_{t} d x
\end{gathered}
$$

Usando a hipótese $\rho>1$, vemos que $x \mapsto|x|^{\rho}$ é diferenciável. Assim,

$$
\frac{1}{2} \frac{d}{d t} \int_{\Omega}\left|u_{t}\right|^{\rho} w_{t}^{2} d x=\int_{\Omega}\left|u_{t}\right|^{\rho} w_{t t} w_{t} d x+\frac{\rho}{2} \int_{\Omega}\left|u_{t}\right|^{\rho-2} u_{t} u_{t t} w_{t}^{2} d x
$$


e, portanto,

$$
\begin{aligned}
\frac{d}{d t} F(t)= & \frac{\rho}{2} \int_{\Omega}\left|u_{t}\right|^{\rho-2} u_{t} u_{t t} w_{t}^{2} d x-\int_{\Omega}\left(\left|u_{t}\right|^{\rho}-\left|v_{t}\right|^{\rho}\right) v_{t t} w_{t} d x \\
& -\int_{\Omega}(f(u)-f(v)) w_{t} d x-\gamma\left\|\nabla w_{t}\right\|_{2}^{2}-\left(\zeta, \zeta_{s}\right)_{\mathcal{M}}+\int_{\Omega}\left(h_{1}-h_{2}\right) w_{t} d x .
\end{aligned}
$$

Neste momento, vamos estimar o lado direito da igualdade acima. Como $\rho>1 \mathrm{e}$

$$
\rho>0 \text { se } N=1,2 \text { e } 1<\rho \leq \frac{2}{N-2} \text { se } N \geq 3
$$

por hipótese em (2.6), então $N=1,2,3$. Logo $H_{0}^{1}(\Omega) \subset L^{6}(\Omega)$. Observando que

$$
\frac{\rho-1}{2(\rho+1)}+\frac{1}{\rho+1}+\frac{1}{2}=1
$$

obtemos da desigualdade de Hölder generalizada que

$$
\begin{aligned}
\frac{\rho}{2} \int_{\Omega}\left|u_{t}\right|^{\rho-1} u_{t t} w_{t}^{2} d x & \leq \frac{\rho}{2}\left\|u_{t}\right\|_{2(\rho+1)}^{\rho-1}\left\|u_{t t}\right\|_{\rho+1}\left\|w_{t}\right\|_{4}^{2} \\
& \leq C_{R T}\left\|\nabla u_{t t}\right\|_{2}\left\|\nabla w_{t}\right\|_{2}^{2} .
\end{aligned}
$$

Notemos que, usando o Teorema do Valor Médio com $0 \leq \theta \leq 1$ e a desigualdade $(a+b)^{k} \leq 2^{k}\left(a^{k}+b^{k}\right)$, para $a, b, k>0$, obtemos:

$$
\begin{aligned}
\left.|| u_{t}\right|^{\rho}-\left|v_{t}\right|^{\rho} \mid & \leq \rho|(1-\theta)| u_{t}|-\theta| v_{t}||^{\rho-1}\left|u_{t}-v_{t}\right| \\
& \leq \rho|| u_{t}|+| v_{t}||^{\rho-1}\left|w_{t}\right| \\
& \leq\left.\rho 2^{\rho-1}|| u_{t}\right|^{\rho-1}+\left|v_{t}\right|^{\rho-1}|| w_{t} \mid .
\end{aligned}
$$

Assim,

$$
\begin{aligned}
-\int_{\Omega}\left(\left|u_{t}\right|^{\rho}-\left|v_{t}\right|^{\rho}\right)\left|v_{t t}\right|\left|w_{t}\right| d x & \leq\left.\int_{\Omega}|| u_{t}\right|^{\rho}-\left|v_{t}\right|^{\rho}|| v_{t t}|| w_{t} \mid d x \\
& \leq \int_{\Omega}\left(\left.\rho 2^{\rho-1}|| u_{t}\right|^{\rho-1}+\left|v_{t}\right|^{\rho-1} \| w_{t}|| v_{t t}|| w_{t} \mid\right) d x \\
& =\rho 2^{\rho-1}\left(\int_{\Omega}\left|u_{t}\right|^{\rho-1}\left|v_{t t}\right|\left|w_{t}\right|^{2} d x+\int_{\Omega}\left|v_{t}\right|^{\rho-1}\left|v_{t t}\right|\left|w_{t}\right|^{2} d x\right) \\
& \leq \rho 2^{\rho-1}\left(\left\|u_{t}\right\|_{2(\rho+1)}^{\rho-1}+\left\|v_{t}\right\|_{2(\rho+1)}^{\rho-1}\right)\left\|v_{t t}\right\|_{\rho+1}\left\|w_{t}\right\|_{4}^{2} \\
& \leq C_{R T}\left\|\nabla v_{t t}\right\|_{2}\left\|\nabla w_{t}\right\|_{2}^{2} .
\end{aligned}
$$

Os termos restantes são fáceis de estimar. De fato,

$$
\begin{aligned}
-\int_{\Omega}(f(u)-f(v)) w_{t} d x & \leq C_{R T}\left(1+\|u\|_{2(p+1)}^{p}+\|v\|_{2(p+1)}^{p}\right)\|w\|_{2(p+1)}\left\|w_{t}\right\|_{2} \\
& \leq C_{R T} F(t)
\end{aligned}
$$


e como em (2.23),

$$
-\left(\zeta^{t}, \zeta_{s}^{t}\right)_{\mathcal{M}}=\frac{1}{2} \int_{0}^{\infty} \mu^{\prime}(s)\left\|\nabla \zeta^{t}(s)\right\|_{2}^{2} d s \leq 0
$$

Finalmente,

$$
\int_{\Omega}\left(h_{1}-h_{2}\right) w_{t} d x \leq\left\|h_{1}-h_{2}\right\|_{2}^{2}+C_{R T} F(t) .
$$

Assim, para algum $C_{1}>0$ suficientemente grande, concluímos que

$$
\frac{d}{d t} F(t) \leq\left\|h_{1}-h_{2}\right\|_{2}^{2}+C_{1}\left(1+\left\|\nabla u_{t t}(t)\right\|_{2}+\left\|\nabla v_{t t}(t)\right\|_{2}\right) F(t) .
$$

Integrando de 0 a $t$ temos

$$
\begin{aligned}
F(t) \leq & F(0)+\int_{0}^{t}\left\|h_{1}-h_{2}\right\|_{2}^{2} d s+\int_{0}^{t} C_{1}\left(1+\left\|\nabla u_{t t}(s)\right\|_{2}+\left\|\nabla v_{t t}(s)\right\|_{2}\right) F(s) d s \\
\leq & F(0)+\left\|h_{1}-h_{2}\right\|_{2}^{2} T \\
& +\left(\int_{0}^{t} C_{1}\left(1+\left\|\nabla u_{t t}(s)\right\|_{2}+\left\|\nabla v_{t t}(s)\right\|_{2}\right)^{2} d s\right)^{\frac{1}{2}}\left(\int_{0}^{t} F^{2}(s) d s\right)^{\frac{1}{2}} .
\end{aligned}
$$

De (2.25) temos

$$
\int_{0}^{T} C_{1}\left(1+\left\|\nabla u_{t t}(s)\right\|_{2}^{2}+\left\|\nabla v_{t t}(s)\right\|_{2}^{2}\right) d s \leq C_{R T}
$$

Logo,

$$
F^{2}(t) \leq 2 A^{2}+C_{R T} \int_{0}^{t} F^{2}(s) d s, \quad \forall t \in[0, T]
$$

onde

$$
A=\left(F(0)+\left\|h_{1}-h_{2}\right\|_{2}^{2} T\right) .
$$

Assim, utilizando a desigualdade de Gronwall,

$$
F^{2}(t) \leq 2 A^{2} e^{C_{R T} t}
$$

isto é,

$$
F(t) \leq \sqrt{2}\left(F(0)+\left\|h_{1}-h_{2}\right\|_{2}^{2} T\right) e^{\frac{C_{R T} T}{2} T}, \quad \forall t \in[0, T] .
$$

Usando (2.29), obtemos

$$
F(0) \leq C_{R T}\left\|\left(w_{0}, w_{1}, \zeta_{0}\right)\right\|_{\mathcal{H}}^{2}
$$

e, portanto,

$$
\begin{aligned}
& \|u(t)-v(t)\|_{H_{0}^{1}}^{2}+\left\|u_{t}(t)-v_{t}(t)\right\|_{H_{0}^{1}}^{2}+\left\|\eta^{t}-\xi^{t}\right\|_{\mathcal{M}}^{2} \\
& \quad \leq C_{R T}\left(\left\|u_{0}-v_{0}\right\|_{H_{0}^{1}}^{2}+\left\|u_{1}-v_{1}\right\|_{H_{0}^{1}}^{2}+\left\|\eta_{0}-\xi_{0}\right\|_{\mathcal{M}}^{2}+\left\|h_{1}-h_{2}\right\|_{2}^{2}\right),
\end{aligned}
$$

para todo $0 \leq t \leq T$. Isto mostra que as soluções de (2.2)-(2.5) dependem continuamente dos dados iniciais. Em particular, o problema possui unicidade.

Portanto, o item (ii) do Teorema 2.2 está provado. 


\subsection{Prova do teorema: estabilidade exponencial}

Usaremos algumas idéias de Messaoudi \& Tatar [33]. Como eles não estudam o problema de memória com história, precisamos fazer algumas adaptações para se adequar ao nosso caso. Consideremos os funcionais

$$
\begin{gathered}
\psi(t)=\frac{1}{\rho+1} \int_{\Omega}\left|u_{t}(t)\right|^{\rho} u_{t}(t) u(t) d x-\int_{\Omega} \Delta u_{t}(t) u(t) d x \\
\chi(t)=\int_{\Omega} \Delta u_{t}(t)\left(\int_{0}^{\infty} \mu(s) \eta^{t}(s) d s\right) d x-\frac{1}{\rho+1} \int_{\Omega}\left|u_{t}(t)\right|^{\rho} u_{t}(t)\left(\int_{0}^{\infty} \mu(s) \eta^{t}(s) d s\right) d x
\end{gathered}
$$

e

$$
\mathcal{L}(t)=M E(t)+\varepsilon \psi(t)+\chi(t)
$$

onde $\varepsilon>0$ e $M>0$ serão fixados posteriormente.

Antes de prosseguirmos, ao considerarmos as soluções fracas, as integrais em $\psi(t)$ são entendidas como dualidade entre $H_{0}^{1}$ e $H^{-1}$. Esta observação foi primeiramente colocada em Cavalcanti et al [8]. Outra maneira de justificar os cálculos necessários é considerar as soluções aproximadas e depois passar ao limite.

Notemos que $E(t)$ é decrescente, pois da estimativa (2.24), passando ao limite, temos

$$
E^{\prime}(t)+\gamma\left\|\nabla u_{t}(t)\right\|_{2}^{2}=\frac{1}{2} \int_{\Omega}\left(\int_{0}^{\infty} \mu^{\prime}(s)\left|\nabla \eta^{t}(s)\right|^{2} d s\right) d x \leq 0 .
$$

Vamos tomar, $C_{s}$ e $C_{0}$ como constantes positivas que dependem das constantes de imersões de Sobolev e dos dados iniciais respectivamente.

Lema 2.4. Para $M>0$ suficientemente grande, existem constantes $\beta_{1}, \beta_{2}>0$ tais que

$$
\beta_{1} E(t) \leq \mathcal{L}(t) \leq \beta_{2} E(t), \quad t \geq 0,
$$

para algum $0<\varepsilon \leq 1$.

Demonstração. Primeiramente, notemos que de (2.30),

$$
|\psi(t)| \leq \frac{1}{\rho+1}\left\|u_{t}\right\|_{2(\rho+1)}^{\rho+1}\|u\|_{2}+\frac{1}{2}\left\|\nabla u_{t}\right\|_{2}^{2}+\frac{1}{2}\|\nabla u\|_{2}^{2} .
$$

Então, da imersão de Sobolev e de $E(t)$ ser decrescente,

$$
\begin{aligned}
\frac{1}{\rho+1}\left\|u_{t}\right\|_{2(\rho+1)}^{\rho+1}\|u\|_{2} & \leq \frac{1}{2(\rho+1)}\left\|u_{t}\right\|_{2(\rho+1)}^{2(\rho+1)}+\frac{1}{2(\rho+1)}\|u\|_{2}^{2} \\
& \leq \frac{1}{2(\rho+1)}\left\|\nabla u_{t}\right\|_{2}^{2 \rho+2}+C_{s}\|\nabla u\|_{2}^{2} \\
& \leq C_{s} E(0)^{\rho}\left\|\nabla u_{t}\right\|_{2}^{2}+C_{s}\|\nabla u\|_{2}^{2}
\end{aligned}
$$


Portanto,

$$
|\psi(t)| \leq C_{0} E(t)
$$

Por outro lado,

$$
\chi(t)=\int_{0}^{\infty} \mu(s)\left(\int_{\Omega} \Delta u_{t}(t) \eta^{t}(s) d x\right) d s-\int_{0}^{\infty} \mu(s)\left(\frac{1}{\rho+1} \int_{\Omega}\left|u_{t}(t)\right|^{\rho} u_{t}(t) \eta^{t}(s) d x\right) d s .
$$

Notando que

$$
\left|\int_{\Omega} \Delta u_{t}(t) \eta^{t}(s) d x\right| \leq \frac{1}{2}\left\|\nabla u_{t}(t)\right\|_{2}^{2}+\frac{1}{2}\left\|\eta^{t}\right\|_{\mathcal{M}}^{2}
$$

e

$$
\left.\frac{1}{\rho+1}\left|\int_{\Omega}\right| u_{t}(t)\right|^{\rho} u_{t}(t) \eta^{t}(s) d x \mid \leq C_{s} E(0)^{\rho}\left\|\nabla u_{t}(t)\right\|_{2}^{2}+C_{s}\left\|\nabla \eta^{t}(s)\right\|_{2}^{2},
$$

então

$$
|\chi(t)| \leq k_{0} C_{0} E(t)
$$

onde $k_{0}$ foi definida em (2.11). Logo, para algum $C_{2}>0$, temos

$$
|\varepsilon \psi(t)+\chi(t)| \leq C_{2} E(t), \quad 0<\varepsilon \leq 1
$$

Tomando $M>C_{2}$, obtemos (2.33) com $\beta_{1}=M-C_{2}$ e $\beta_{2}=M+C_{2}$.

Lema 2.5. Existe $C_{3}>0$, dependente de $E(0)$, tal que

$$
\begin{aligned}
\psi^{\prime}(t) \leq & -E(t)-\frac{1}{4}\|\nabla u(t)\|_{2}^{2}+C_{3}\left\|\nabla u_{t}(t)\right\|_{2}^{2} \\
& -C_{3} \int_{0}^{\infty} \mu^{\prime}(s)\left\|\nabla \eta^{t}(s)\right\|_{2}^{2} d s, \quad \forall t \geq 0 .
\end{aligned}
$$

Demonstração. Da definição de $\psi(t)$,

$$
\psi^{\prime}(t)=\int_{\Omega}\left(\left|u_{t}\right|^{\rho} u_{t t}-\Delta u_{t t}\right) u d x+\frac{1}{\rho+1}\left\|u_{t}\right\|_{\rho+2}^{\rho+2}+\left\|\nabla u_{t}\right\|_{2}^{2} .
$$

Usando a equação (2.2), observamos que

$$
\begin{aligned}
\int_{\Omega}\left(\left|u_{t}\right|^{\rho} u_{t t}-\Delta u_{t t}\right) u d x= & -\|\nabla u\|_{2}^{2}+\int_{0}^{\infty} \mu(s)\left(\int_{\Omega} \Delta \eta^{t}(s) u(t) d x\right) d s \\
& +\gamma \int_{\Omega} \Delta u_{t} u d x-\int_{\Omega} f(u) u d x .
\end{aligned}
$$

Como

$$
\int_{0}^{\infty} \mu(s)\left(\int_{\Omega} \Delta \eta^{t}(s) u(t) d x\right) d s \leq \frac{1}{8}\|\nabla u\|_{2}^{2}+2 k_{0}\left\|\eta^{t}\right\|_{\mathcal{M}}^{2}
$$


$\mathrm{e}$

$$
\gamma \int_{\Omega} \Delta u_{t} u d x \leq \frac{1}{8}\|\nabla u\|_{2}^{2}+2 \gamma^{2}\left\|\nabla u_{t}\right\|_{2}^{2}
$$

então

$$
\psi^{\prime}(t) \leq-\frac{3}{4}\|\nabla u\|_{2}^{2}+\left(1+2 \gamma^{2}\right)\left\|\nabla u_{t}\right\|_{2}^{2}+\frac{1}{\rho+1}\left\|u_{t}\right\|_{\rho+2}^{\rho+2}+2 k_{0}\left\|\eta^{t}\right\|_{\mathcal{M}}^{2}-\int_{\Omega} f(u) u d x .
$$

Subtraindo e somando $E(t)$ e utilizando (2.9) temos

$$
\psi^{\prime}(t) \leq-E(t)-\frac{1}{4}\|\nabla u\|_{2}^{2}+\left(\frac{3}{2}+2 \gamma^{2}\right)\left\|\nabla u_{t}(t)\right\|_{2}^{2}+\frac{2}{\rho+1}\left\|u_{t}\right\|_{\rho+2}^{\rho+2}+\left(\frac{1}{2}+2 k_{0}\right)\left\|\eta^{t}\right\|_{\mathcal{M}}^{2}
$$

Logo, de

$$
\left\|u_{t}(t)\right\|_{\rho+2}^{\rho+2} \leq C_{s} E(0)^{\frac{\rho}{2}}\left\|\nabla u_{t}(t)\right\|_{2}^{2}
$$

$\mathrm{e}$

$$
\left\|\eta^{t}\right\|_{\mathcal{M}}^{2} \leq-\frac{1}{k_{1}} \int_{0}^{\infty} \mu^{\prime}(s)\left\|\nabla \eta^{t}(s)\right\|_{2}^{2} d s
$$

concluímos que (2.35) vale para algum $C_{3}>0$.

Lema 2.6. Dado $\delta_{0}>0$ existe $C_{4}>0$, dependente de $E(0)$, tal que

$$
\chi^{\prime}(t) \leq-\frac{k_{0}}{4}\left\|\nabla u_{t}(t)\right\|_{2}^{2}+\delta_{0}\|\nabla u(t)\|_{2}^{2}-C_{4} \int_{0}^{\infty} \mu^{\prime}(s)\left\|\nabla \eta^{t}(s)\right\|_{2}^{2} d s .
$$

Demonstração. Da definição de $\chi(t)$,

$$
\chi^{\prime}(t)=I_{1}+I_{2},
$$

onde

$$
I_{1}=\int_{\Omega}\left(-\left|u_{t}\right|^{\rho} u_{t t}+\Delta u_{t t}\right)\left(\int_{0}^{\infty} \mu(s) \eta(s) d s\right) d x
$$

e

$$
I_{2}=\int_{\Omega}\left(-\frac{\left|u_{t}\right|^{\rho} u_{t}}{\rho+1}+\Delta u_{t}\right)\left(\int_{0}^{\infty} \mu(s) \eta_{t}(s) d s\right) d x
$$

Da equação (2.2) obtemos

$$
I_{1}=\int_{\Omega}\left(-\Delta u-\int_{0}^{\infty} \mu(s) \Delta \eta(s) d s-\gamma \Delta u_{t}+f(u)\right)\left(\int_{0}^{\infty} \mu(s) \eta(s) d s\right) d x .
$$

Assim, temos as seguintes estimativas:

$$
\int_{\Omega}-\Delta u(t)\left(\int_{0}^{\infty} \mu(s) \eta^{t}(s) d s\right) d x \leq \delta\|\nabla u(t)\|_{2}^{2}+\frac{k_{0}}{4 \delta}\left\|\eta^{t}\right\|_{\mathcal{M}}^{2}
$$




$$
\begin{gathered}
\int_{\Omega}-\gamma \Delta u_{t}(t)\left(\int_{0}^{\infty} \mu(s) \eta^{t}(s) d s\right) d x \leq \frac{k_{0}}{4}\left\|\nabla u_{t}(t)\right\|_{2}^{2}+\left\|\eta^{t}\right\|_{\mathcal{M}}^{2} \\
\int_{\Omega} f(u(t))\left(\int_{0}^{\infty} \mu(s) \eta^{t}(s) d s\right) d x \leq \delta C_{s} E(0)^{p} k_{0}\|\nabla u(t)\|_{2}^{2}+\frac{1}{4 \delta}\left\|\eta^{t}\right\|_{\mathcal{M}}^{2} \\
\int_{\Omega}-\left(\int_{0}^{\infty} \mu(s) \Delta \eta(s) d s\right)\left(\int_{0}^{\infty} \mu(s) \eta(s) d s\right) d x \\
=\int_{\Omega} \sum_{j=1}^{N}\left(\int_{0}^{\infty} \mu(s) \frac{\partial \eta}{\partial x_{j}} d s\right)^{2} d x \\
\leq \int_{\Omega} \sum_{j=1}^{N} k_{0}\left(\int_{0}^{\infty} \mu(s)\left|\frac{\partial \eta}{\partial x_{j}}\right|^{2} d s\right) d x \\
\leq k_{0}\left\|\eta^{t}\right\|_{\mathcal{M}}^{2} .
\end{gathered}
$$

Portanto, dado $\delta_{0}>0$ pequeno, existe $C^{\prime}>0$ tal que

$$
\begin{aligned}
I_{1} & \leq \delta_{0}\|\nabla u(t)\|_{2}^{2}+\frac{k_{0}}{4}\left\|\nabla u_{t}(t)\right\|_{2}^{2}+C^{\prime}\left\|\eta^{t}\right\|_{\mathcal{M}}^{2} \\
& \leq \delta_{0}\|\nabla u(t)\|_{2}^{2}+\frac{k_{0}}{4}\left\|\nabla u_{t}(t)\right\|_{2}^{2}-\frac{C^{\prime}}{k_{1}} \int_{0}^{\infty} \mu^{\prime}(s)\left\|\nabla \eta^{t}(s)\right\|_{2}^{2} d s .
\end{aligned}
$$

Agora, vamos estimar $I_{2}$. De (2.3),

$$
\begin{aligned}
\int_{0}^{\infty} \mu(s) \eta_{t}^{t}(s) d s & =-\int_{0}^{\infty} \mu(s) \eta_{s}^{t}(s) d s+\int_{0}^{\infty} \mu(s) u_{t}(t) d s \\
& =\int_{0}^{\infty} \mu^{\prime}(s) \eta^{t}(s) d s+k_{0} u_{t}(t) .
\end{aligned}
$$

Então,

$$
\begin{aligned}
I_{2}= & -k_{0}\left\|\nabla u_{t}(t)\right\|_{2}^{2}-\frac{k_{0}}{\rho+1}\left\|u_{t}(t)\right\|_{\rho+2}^{\rho+2}+\int_{0}^{\infty} \mu^{\prime}(s)\left(\int_{\Omega} \Delta u_{t}(t) \eta^{t}(s) d x\right) d s \\
& +\frac{1}{\rho+1} \int_{0}^{\infty} \mu^{\prime}(s)\left(\int_{\Omega}-\left|u_{t}(t)\right|^{\rho} u_{t}(t) \eta^{t}(s) d x\right) d s .
\end{aligned}
$$

Observemos que, para alguma constante $C^{\prime \prime}>0$,

$$
\begin{aligned}
\int_{0}^{\infty} \mu^{\prime}(s)\left(\int_{\Omega} \Delta u_{t}(t) \eta^{t}(s) d x\right) d s & \leq-\int_{0}^{\infty} \mu^{\prime}(s)\left\|\nabla u_{t}(t)\right\|_{2}\left\|\nabla \eta^{t}(s)\right\|_{2} d s \\
& \leq \frac{k_{0}}{4}\left\|\nabla u_{t}(t)\right\|_{2}^{2}-C^{\prime \prime} \int_{0}^{\infty} \mu^{\prime}(s)\left\|\nabla \eta^{t}(s)\right\|_{2}^{2} d s
\end{aligned}
$$

e

$$
\begin{aligned}
\frac{1}{\rho+1} \int_{0}^{\infty} \mu^{\prime}(s) & \left(\int_{\Omega}-\left|u_{t}(t)\right|^{\rho} u_{t}(t) \eta^{t}(s) d x\right) d s \\
\leq & \frac{k_{0}}{4}\left\|\nabla u_{t}(t)\right\|_{2}^{2}-C^{\prime \prime} \int_{0}^{\infty} \mu^{\prime}(s)\left\|\nabla \eta^{t}(s)\right\|_{2}^{2} d s
\end{aligned}
$$


Logo,

$$
I_{2} \leq-\frac{k_{0}}{2}\left\|\nabla u_{t}(t)\right\|_{2}^{2}-2 C^{\prime \prime} \int_{0}^{\infty} \mu^{\prime}(s)\left\|\nabla \eta^{t}(s)\right\|_{2}^{2} d s,
$$

que juntamente com (2.37) mostram que (2.36) vale para algum $C_{4}=C_{4}\left(\delta_{0}, C^{\prime}, C^{\prime \prime}\right)$.

Prova do Teorema 2.2 (iii): Primeiramente, vamos fixar $\varepsilon>0$ tal que $\varepsilon C_{3}<k_{0} / 4$. Então, tomando $\delta_{0}>0$ tal que $\delta_{0}<\varepsilon / 4$, os Lemas 2.5 e 2.6 asseguram que

$$
\varepsilon \psi^{\prime}(t)+\chi^{\prime}(t) \leq-\varepsilon E(t)-C_{5} \int_{0}^{\infty} \mu^{\prime}(s)\left\|\nabla \eta^{t}(s)\right\|_{2}^{2} d s,
$$

onde $C_{5}=\varepsilon C_{3}+C_{4}$. Escolhendo $M>2 C_{5}$, obtemos de (2.32) que

$$
M E^{\prime}(t)+\varepsilon \psi^{\prime}(t)+\chi^{\prime}(t) \leq-\varepsilon E(t)
$$

isto é,

$$
\mathcal{L}^{\prime}(t) \leq-\varepsilon E(t)
$$

Agora, podemos escolher $M>0$ suficiente grande para que possamos aplicar o Lema 2.4. Então, combinando (2.38) com (2.33), obtemos (2.16) usando o seguinte raciocínio: De (2.33) temos

$$
\mathcal{L}^{\prime}(t) \leq-\frac{\varepsilon}{\beta_{2}} \mathcal{L}(t)
$$

A desigualdade de Gronwall implica que

$$
\mathcal{L}(t) \leq \mathcal{L}(0) e^{-\frac{\varepsilon}{\beta_{2}} t}
$$

Usando (2.33) novamente, obtemos

$$
\beta_{1} E(t) \leq \mathcal{L}(t) \leq \beta_{2} E(0) e^{-\frac{\varepsilon}{\beta_{2}} t}
$$

ou seja, a estimativa de energia (2.16) vale $\operatorname{com} \nu=\varepsilon / \beta_{2}$ e $K=\beta_{2} / \beta_{1}$. 


$\frac{1}{3}$

\section{Existência de um atrator global}

Neste capítulo, provamos a existência de um atrator global para o problema (2.2)-(2.5). Observamos que do Teorema 2.2, o operador solução

$$
S(t): \mathcal{H} \rightarrow \mathcal{H}, \quad S(t)\left(u_{0}, v_{0}, \eta_{0}\right)=\left(u(t), u_{t}(t), \eta^{t}\right), \quad t \geq 0,
$$

onde $\left(u(t), u_{t}(t), \eta^{t}\right)$ é uma solução fraca que corresponde aos dados iniciais $\left(u_{0}, v_{0}, \eta_{0}\right)$, define um semigrupo de evolução em $\mathcal{H}$. De fato, da unicidade de solução, $S(t)$ satisfaz as propriedades de semigrupo

$$
S(0)=I \quad \text { e } \quad S(t+s)=S(t) S(s), \quad s, t \geq 0 .
$$

Também, a dependência contínua dos dados iniciais em $\mathcal{H}$ e (2.15) implicam que $S(t)$ é fortemente contínuo sobre $\mathcal{H}$. Assim, $S(t)$ é um semigrupo $C^{0}$ de $\mathcal{H}$ e, consequentemente, o problema (2.2)-(2.5) pode ser visto como um sistema dinâmico de dimensão infinita nãolinear $(\mathcal{H}, S(t))$.

Teorema 3.1. Sob as hipóteses do Teorema 2.2, com $h \in L^{2}(\Omega)$ e $\gamma>0$, o sistema dinâmico $(\mathcal{H}, S(t))$ correspondente ao problema (2.2)-(2.5) possui atrator global. 
Observação 3.2. A existência de um atrator global é estabelecida para a equação com dissipação forte $-\gamma \Delta u_{t}$, isto é, $\gamma>0$. Porém, a demonstração não assume explicitamente $\rho>1$.

\subsection{Prova do teorema}

\subsubsection{Conjunto absorvente}

Lema 3.3. Sob as hipóteses do Teorema 3.1, o sistema dinâmico $(\mathcal{H}, S(t))$ associado ao problema (2.2)-(2.5) possui um conjunto absorvente limitado.

Demonstração. Consideremos o funcional

$$
\mathcal{J}(t)=E(t)+\varepsilon \psi(t), \quad \varepsilon>0
$$

onde $\psi(t)$ é o mesmo funcional definido em (2.30).

Etapa 1. Seja $\lambda_{1}>0$ o primeiro autovalor de $-\Delta$ em $H_{0}^{1}(\Omega)$. Então,

$$
\frac{1}{4}\left(\|\nabla u(t)\|_{2}^{2}+\left\|\nabla u_{t}(t)\right\|_{2}^{2}+\left\|\eta^{t}\right\|_{\mathcal{M}}^{2}\right) \leq E(t)+\frac{1}{\lambda_{1}}\|h\|_{2}^{2}
$$

De fato, como

$$
\int_{\Omega} h u d x \leq \frac{1}{4}\|\nabla u\|_{2}^{2}+\frac{1}{\lambda_{1}}\|h\|_{2}^{2}
$$

obtemos

$$
E(t) \geq \frac{1}{\rho+2}\left\|u_{t}\right\|_{\rho+2}^{\rho+2}+\frac{1}{4}\|\nabla u\|_{2}^{2}+\frac{1}{2}\left\|\nabla u_{t}\right\|_{2}^{2}+\frac{1}{2}\left\|\eta^{t}\right\|_{\mathcal{M}}^{2}+\int_{\Omega} \hat{f}(u) d x-\frac{1}{\lambda_{1}}\|h\|_{2}^{2} .
$$

Logo, usando (2.9), temos (3.2).

Etapa 2. Existe $\varepsilon_{0}>0$, que depende dos dados iniciais, tal que

$$
\frac{1}{2} E(t)-\frac{1}{2 \lambda_{1}}\|h\|_{2}^{2} \leq \mathcal{J}(t) \leq \frac{3}{2} E(t)+\frac{1}{2 \lambda_{1}}\|h\|_{2}^{2}, \quad t \geq 0, \quad \varepsilon \in\left(0, \varepsilon_{0}\right]
$$

Para provarmos isto, primeiramente observamos que

$$
\int_{\Omega}\left|u_{t}\right|^{\rho+1} u d x \leq \frac{1}{2}\left(\left\|u_{t}\right\|_{\rho+2}^{2(\rho+1)}+\|u\|_{\rho+2}^{2}\right) \leq C_{s} E(0)^{\rho}\left\|\nabla u_{t}\right\|_{2}^{2}+C_{s}\|\nabla u\|_{2}^{2} .
$$

Então, de (3.2),

$$
\begin{aligned}
|\psi(t)| & \leq C_{0}\left(\|\nabla u(t)\|_{2}^{2}+\left\|\nabla u_{t}(t)\right\|_{2}^{2}\right) \\
& \leq 4 C_{0}\left(E(t)+\frac{1}{\lambda_{1}}\|h\|_{2}^{2}\right) .
\end{aligned}
$$


Tomando $\varepsilon_{0}=\frac{1}{8 C_{0}}$ temos

$$
|\varepsilon \psi(t)| \leq \frac{1}{2} E(t)+\frac{1}{2 \lambda_{1}}\|h\|_{2}^{2}, \quad \forall \varepsilon \in\left(0, \varepsilon_{0}\right]
$$

que implica em (3.3).

Etapa 3. Existe $C_{6}>0$, que depende dos dados iniciais, tal que

$$
\psi^{\prime}(t) \leq-E(t)+C_{6}\left\|\nabla u_{t}(t)\right\|_{2}^{2}-C_{6} \int_{0}^{\infty} \mu^{\prime}(s)\left\|\nabla \eta^{t}(s)\right\|_{2}^{2} d s, \quad \forall t \geq 0 .
$$

A prova é a mesma do Lema 2.5 , já que $\int_{\Omega} h u d x$ é cancelado nos cálculos.

Etapa 4. Conclusão. Tendo em mente (2.32) $\operatorname{com} \gamma>0$, escolhemos $\varepsilon_{1}=\min \left\{\frac{\gamma}{C_{6}}, \frac{1}{2 C_{6}}\right\}, \mathrm{o}$ qual é diferente de zero, pois assumimos $\gamma>0$. Então, (3.4) implica que

$$
\mathcal{J}^{\prime}(t)=E^{\prime}(t)+\varepsilon \psi^{\prime}(t) \leq-\varepsilon E(t), \quad t \geq 0, \quad \varepsilon \in\left(0, \varepsilon_{1}\right] .
$$

Tomemos $\varepsilon \leq \min \left\{\varepsilon_{0}, \varepsilon_{1}\right\}$. Usando (3.3) vemos que

$$
\mathcal{J}^{\prime}(t) \leq-\frac{2 \varepsilon}{3} \mathcal{J}(t)+\frac{\varepsilon}{3 \lambda_{1}}\|h\|_{2}^{2}
$$

e, da desigualdade de Gronwall, temos

$$
\begin{aligned}
\mathcal{J}(t) & \leq \mathcal{J}(0) e^{-\frac{2 \varepsilon}{3} t}+\frac{\varepsilon}{3 \lambda_{1}}\|h\|_{2}^{2} \int_{0}^{t} e^{-\frac{2 \varepsilon}{3}(t-s)} d s \\
& =\left(\mathcal{J}(0)-\frac{1}{2 \lambda_{1}}\|h\|_{2}^{2}\right) e^{-\frac{2 \varepsilon}{3} t}+\frac{1}{2 \lambda_{1}}\|h\|_{2}^{2} .
\end{aligned}
$$

Usando novamente (3.3),

$$
\frac{1}{2} E(t) \leq \frac{3}{2} E(0) e^{-\frac{2 \varepsilon}{3} t}+\frac{1}{\lambda_{1}}\|h\|_{2}^{2}
$$

Portanto, de (3.2), deduzimos que

$$
\left\|\left(u(t), u_{t}(t), \eta^{t}\right)\right\|_{\mathcal{H}}^{2} \leq 12 E(0) e^{-\frac{2 \varepsilon}{3} t}+\frac{12}{\lambda_{1}}\|h\|_{2}^{2} .
$$

Isto nos mostra que qualquer bola fechada $\bar{B}(0, R) \operatorname{com} R>\sqrt{\frac{12}{\lambda_{1}}}\|h\|_{2}$ é um conjunto absorvente limitado de $(\mathcal{H}, S(t))$. 


\subsubsection{Desigualdade de estabilização}

Lema 3.4 (Estabilização). Sob as hipóteses do Teorema 3.1, dado um conjunto limitado $B \subset \mathcal{H}$, sejam $z^{1}=\left(u, u_{t}, \eta\right) e z^{2}=\left(v, v_{t}, \xi\right)$ duas soluções fracas do problema $(2.2)-(2.5)$ tais que $z^{1}(0)=\left(u_{0}, u_{1}, \eta_{0}\right)$ e $z^{2}(0)=\left(v_{0}, v_{1}, \xi_{0}\right)$ pertencem a B. Então,

$$
\begin{aligned}
\left\|z^{1}(t)-z^{2}(t)\right\|_{\mathcal{H}}^{2} \leq & C_{B} e^{-\nu t}+C_{B} \int_{0}^{t} e^{-\nu(t-s)}\left(1+\left\|\nabla u_{t t}(s)\right\|_{2}+\left\|\nabla v_{t t}(s)\right\|_{2}\right) \\
& \times\left(\|w(s)\|_{2}+\left\|w_{t}(s)\right\|_{2}\right) d s
\end{aligned}
$$

onde $\nu$ e $C_{B}$ são constantes positivas que dependem de $B$ e $w=u-v$.

Demonstração. Sejam $w=u-v$ e $\zeta=\eta-\xi$. Então, $(w, \zeta)$ satisfaz

$$
\begin{aligned}
& -\Delta w-\Delta w_{t t}-\int_{0}^{\infty} \mu(s) \Delta \zeta^{t}(s) d s=\gamma \Delta w_{t}-\left|u_{t}\right|^{\rho} u_{t t}+\left|v_{t}\right|^{\rho} v_{t t}-f(u)+f(v) \\
& \zeta_{t}=-\zeta_{s}+w_{t}
\end{aligned}
$$

com condição inicial

$$
w(0)=u_{0}-v_{0}, \quad w_{t}(0)=u_{1}-v_{1}, \quad \eta^{0}=\zeta_{0}-\xi_{0} .
$$

Agora, consideremos o funcional

$$
G(t)=\frac{1}{2}\|\nabla w(t)\|_{2}^{2}+\frac{1}{2}\left\|\nabla w_{t}(t)\right\|_{2}^{2}+\frac{1}{2}\left\|\zeta^{t}\right\|_{\mathcal{M}}^{2}
$$

e sua pertubação

$$
\mathcal{G}(t)=G(t)+\varepsilon \phi(t),
$$

onde

$$
\phi(t)=-\int_{\Omega} \Delta w_{t}(t) w(t) d x
$$

Dividimos o restante da prova em quatro etapas.

Etapa 1. Notemos que

$$
\frac{1}{2} G(t) \leq \mathcal{G}(t) \leq \frac{3}{2} G(t), \quad t \geq 0, \quad 0<\varepsilon \leq \frac{1}{2} .
$$

Isto segue diretamente das definições de $\phi$ e $G$. 
Etapa 2. Existe uma constante $C_{7}>0$ (dependendo de $B$ ) tal que

$$
\begin{aligned}
G^{\prime}(t) \leq & -\gamma\left\|\nabla w_{t}(t)\right\|_{2}^{2}+\frac{1}{2} \int_{0}^{\infty} \mu^{\prime}(s)\left\|\nabla \zeta^{t}(s)\right\|_{2}^{2} d s \\
& +C_{7}\left(1+\left\|\nabla u_{t t}(t)\right\|_{2}+\left\|\nabla v_{t t}(t)\right\|_{2}\right)\left\|\nabla w_{t}(t)\right\|_{2}^{2}
\end{aligned}
$$

Para provar isto, multiplicamos (3.6) por $w_{t}$ e (3.7) por $\zeta^{t}$. Então, por integração, vemos que

$$
G^{\prime}(t) \leq-\gamma\left\|\nabla w_{t}\right\|_{2}^{2}-\left(\zeta_{t}, \zeta_{s}\right)-\int_{\Omega}\left(\left|u_{t}\right|^{\rho} u_{t t}-\left|v_{t}\right|^{\rho} v_{t t}\right) w_{t} d x-\int_{\Omega}(f(u)-f(v)) w_{t} d x
$$

Como na prova da unicidade,

$$
\begin{gathered}
-\left(\zeta_{t}, \zeta_{s}\right)=\frac{1}{2} \int_{0}^{\infty} \mu^{\prime}(s)\left\|\nabla \zeta^{t}(s)\right\|_{2}^{2} d s \\
-\int_{\Omega}\left(\left|u_{t}\right|^{\rho} u_{t t}-\left|v_{t}\right|^{\rho} v_{t t}\right) w_{t} d x \leq C_{0}\left(\left\|\nabla u_{t t}\right\|_{2}+\left\|\nabla v_{t t}\right\|_{2}\right)\left\|\nabla w_{t}\right\|_{2}^{2} \\
-\int_{\Omega}(f(u)-f(v)) w_{t} d x \leq C_{0}\left\|\nabla w_{t}\right\|_{2}^{2} .
\end{gathered}
$$

Então, vale (3.9).

Etapa 3. Existe uma constante $C_{8}>0$ tal que

$$
\begin{aligned}
\phi^{\prime}(t) \leq & -G(t)+\left(\frac{3}{2}+2 \gamma^{2}\right)\left\|\nabla w_{t}(t)\right\|_{2}^{2}-\left(\frac{1+2 k_{0}}{2 k_{1}}\right) \int_{0}^{\infty} \mu^{\prime}(s)\left\|\nabla \zeta^{t}(s)\right\|_{2}^{2} d s \\
& +C_{8}\left(1+\left\|\nabla u_{t t}(t)\right\|_{2}+\left\|\nabla v_{t t}(t)\right\|_{2}\right)\|\nabla w(t)\|_{2}^{2} .
\end{aligned}
$$

De fato, temos

$$
\begin{aligned}
\phi^{\prime}(t)= & \int_{\Omega}-\Delta w_{t t} w d x+\left\|\nabla w_{t}\right\|_{2}^{2} \\
= & \int_{\Omega}\left(\int_{0}^{\infty} \mu(s) \Delta \zeta(s) d s+\gamma \Delta w_{t}-\left(\left|u_{t}\right|^{\rho} u_{t t}-\left|v_{t}\right|^{\rho} v_{t t}\right)-(f(u)-f(v))\right) w d x \\
& -\|\nabla w\|_{2}^{2}+\left\|\nabla w_{t}\right\|_{2}^{2} .
\end{aligned}
$$

Além disso,

$$
\begin{gathered}
\int_{0}^{\infty} \mu(s)\left(\int_{\Omega} \Delta \zeta(s) w d x\right) d s \leq \frac{1}{4}\|\nabla w\|_{2}^{2}+k_{0}\|\zeta\|_{\mathcal{M}}^{2} \\
\gamma \int_{\Omega} \Delta w_{t} w d x \leq \frac{1}{4}\|\nabla w\|_{2}^{2}+\gamma^{2}\left\|\nabla w_{t}\right\|_{2}^{2},
\end{gathered}
$$


$-\int_{\Omega}\left(\left|u_{t}\right|^{\rho} u_{t t}-\left|v_{t}\right|^{\rho} v_{t t}\right) w d x-\int_{\Omega}(f(u)-f(v)) w d x \leq C_{0}\left(1+\left\|\nabla u_{t t}\right\|_{2}+\left\|\nabla v_{t t}\right\|_{2}\right)\|\nabla w\|_{2}^{2}$.

Portanto,

$$
\phi^{\prime}(t) \leq-\frac{1}{2}\|\nabla w\|_{2}^{2}+\left(1+\gamma^{2}\right)\left\|\nabla w_{t}\right\|_{2}^{2}+k_{0}\|\zeta\|_{\mathcal{M}}^{2}+C_{0}\left(1+\left\|\nabla u_{t t}\right\|_{2}+\left\|\nabla v_{t t}\right\|_{2}\right)\|\nabla w\|_{2}^{2}
$$

Subtraindo e somando $G(t)$ na desigualdade acima, obtemos (3.10).

Etapa 4. Conclusão. Tomemos

$$
\varepsilon<\min \left\{\frac{1}{2}, \frac{3+4 \gamma^{2}}{2 \gamma}, \frac{1+2 k_{0}}{k_{1}}\right\}
$$

Então, (3.8) vale e

$$
\mathcal{G}^{\prime}(t) \leq-\varepsilon G(t)+C_{0}\left(1+\left\|\nabla u_{t t}(t)\right\|_{2}+\left\|\nabla v_{t t}(t)\right\|_{2}\right)\left(\|\nabla w(t)\|_{2}^{2}+\left\|w_{t}(t)\right\|_{2}\right) .
$$

Usando (3.8), vemos que

$$
\mathcal{G}^{\prime}(t) \leq-\frac{2 \varepsilon}{3} \mathcal{G}(t)+m(t)
$$

onde

$$
m(t)=C_{0}\left(1+\left\|\nabla u_{t t}(t)\right\|_{2}+\left\|\nabla v_{t t}(t)\right\|_{2}\right)\left(\|\nabla w(t)\|_{2}^{2}+\left\|w_{t}(t)\right\|_{2}\right) .
$$

Integrando obtemos

$$
\mathcal{G}(t) \leq \mathcal{G}(0) e^{-\frac{2 \varepsilon}{3} t}+\int_{0}^{t} e^{-\frac{2 \varepsilon}{3}(t-s)} m(s) d s .
$$

Usando novamente (3.8) temos

$$
G(t) \leq 3 G(0) e^{-\frac{2 \varepsilon}{3} t}+2 \int_{0}^{t} e^{-\frac{2 \varepsilon}{3}(t-s)} m(s) d s
$$

Como $G(t)=\frac{1}{2}\left\|z^{1}(t)-z^{2}(t)\right\|_{\mathcal{H}}^{2}$, obtemos (3.5) com $\nu=2 \varepsilon / 3$. 


\subsubsection{Compacidade assintótica}

Lema 3.5. O sistema dinâmico associado ao problema (2.2)-(2.5) com $\gamma>0$ é assintoticamente compacto.

Demonstração. Vamos usar o Teorema 1.21. Seja $B$ um subconjunto limitado de $\mathcal{H}$ e positivamente invariante com respeito a $S(t)$. Dado $\epsilon>0$, tomamos $T>0$ tal que $C_{B} e^{-\frac{\nu}{2} T}<\epsilon$, onde $C_{B}$ é dado no Lema 3.4. Afirmamos que existe uma constante $C_{B T}>0$ tal que

$$
\left\|S(T) z_{0}^{1}-S(T) z_{0}^{2}\right\|_{\mathcal{H}} \leq \epsilon+\Phi_{T}\left(z_{0}^{1}, z_{0}^{2}\right), \quad \forall z_{0}^{1}, z_{0}^{2} \in B
$$

com

$$
\Phi_{T}\left(z_{0}^{1}, z_{0}^{2}\right)=C_{B T}\left(\int_{0}^{T}\|u(s)-v(s)\|_{2}^{2}+\left\|u_{t}(s)-v_{t}(s)\right\|_{2}^{2} d s\right)^{\frac{1}{4}} .
$$

Estamos considerando que $z_{1}(t)=\left(u(t), u_{t}(t), \eta^{t}\right)=S(t) z_{0}^{1}$ e $z_{2}(t)=\left(v(t), v_{t}(t), \xi^{t}\right)=$ $S(t) z_{0}^{2}$ são soluções de (2.2)-(2.5) com respeito aos dados iniciais $z_{0}^{1}, z_{0}^{2} \in B$ respectivamente.

Vamos provar (3.11)-(3.12). No contexto do Lema 3.4 temos

$$
\begin{aligned}
\left\|z_{1}(t)-z_{2}(t)\right\|_{\mathcal{H}}^{2} \leq & C_{B} e^{-\nu t}+C_{B}\left(\int_{0}^{T}\left(1+\left\|\nabla u_{t t}(s)\right\|_{2}+\left\|\nabla v_{t t}(s)\right\|_{2}\right)^{2} d s\right)^{\frac{1}{2}} \\
& \times\left(\int_{0}^{T}\left(\|w(s)\|_{2}+\left\|w_{t}(s)\right\|_{2}\right)^{2} d s\right)^{\frac{1}{2}}, \quad 0<t<T .
\end{aligned}
$$

Como $u_{t t}, v_{t t}$ são limitados em $L^{2}\left(0, T ; H_{0}^{1}(\Omega)\right)$, concluímos que existe $C_{B T}>0$ tal que

$$
\left\|z^{1}(T)-z^{2}(T)\right\|_{\mathcal{H}} \leq C_{B} e^{-\frac{\nu}{2} T}+C_{B T}\left(\int_{0}^{T}\|w(s)\|_{2}^{2}+\left\|w_{t}(s)\right\|_{2}^{2} d s\right)^{\frac{1}{4}}
$$

implicando que (3.11)-(3.12) vale.

Resta-nos mostrar que $\phi_{T}$ satisfaz (1.2). De fato, dada uma sequência $\left(z_{0}^{n}\right)=$ $\left(u_{0}^{n}, u_{1}^{n}, \eta_{0}^{n}\right) \in B$, consideremos $S(t)\left(z_{0}^{n}\right)=\left(u^{n}(t), u_{t}^{n}(t), \eta^{n t}\right)$. Como $B$ é positivamente 
invariante $S(t)$, segue que a sequência $\left(u^{n}, u_{t}^{n}, \eta^{n t}\right)$ é uniformemente limitada em $\mathcal{H}$. Em particular,

$$
\left(u^{n}\right) \text { e }\left(u_{t}^{n}\right) \text { são limitadas em } C\left([0, \infty) ; H_{0}^{1}(\Omega)\right) .
$$

Notemos que, como $u_{t t}$ é limitada em $L^{2}\left(0, T ; H_{0}^{1}(\Omega)\right)$, então

$$
\left(u_{t}^{n}\right) \text { e }\left(u_{t t}^{n}\right) \text { são limitadas em } L^{2}\left(0, T ; L^{2}(\Omega)\right)
$$

Devido a imersão compacta $H_{0}^{1}(\Omega) \hookrightarrow \hookrightarrow L^{2}(\Omega)$, passando a uma subsequência se necessário, o Teorema de Simon (Teorema 1.6, Capítulo 1) implica que

$$
\left(u^{n}\right) \text { e }\left(u_{t}^{n}\right) \text { convergem fortemente em } C\left([0, T] ; L^{2}(\Omega)\right)
$$

Portanto, concluímos que

$$
\lim _{n \rightarrow \infty} \lim _{m \rightarrow \infty} \int_{0}^{T}\left\|u^{n}(s)-u^{m}(s)\right\|_{2}^{2}+\left\|u_{t}^{n}(s)-u_{t}^{m}(s)\right\|_{2}^{2} d s=0,
$$

mostrando que vale (1.2). Assim, $(\mathcal{H}, S(t))$ é assintoticamente compacto, uma vez que o sistema é dissipativo.

Prova do Teorema 3.1 Os Lemas 3.3 e 3.5 implicam que $(\mathcal{H}, S(t))$ é um sistema dinâmico dissipativo e assintoticamente compacto. Então, a existência de um atrator global compacto para o problema (2.2)-(2.5) segue do Teorema 1.19. 


\begin{tabular}{l|l|}
\hline \multicolumn{1}{|c|}{} & CарÍtulo \\
\hline & 4 \\
\hline
\end{tabular}

\section{Um modelo de Kirchhoff} viscoelástico: atratores

exponenciais

\subsection{Introdução}

Neste capítulo, estudamos a existência de atratores exponenciais para o sistema correspondente à equação (4). Mais precisamente, seja $\Omega$ um domínio limitado de $\mathbb{R}^{N}$, $N \geq 1$, com fronteira regular $\Gamma=\partial \Omega$. Então, o problema é apresentado em Jorge Silva \& 
Ma [24] na seguinte forma:

$$
u_{t t}+\alpha \Delta^{2} u-\Delta_{p} u-\int_{0}^{\infty} \mu(s) \Delta^{2} u(t-s) d s-\Delta u_{t}+f(u)=h \quad \text { em } \quad \Omega \times \mathbb{R}^{+},
$$

com condições de fronteira

$$
u=\Delta u=0 \quad \text { sobre } \quad \Gamma \times \mathbb{R}
$$

e condições iniciais

$$
u(x, \tau)=u_{0}(x, \tau) \quad \text { e } \quad u_{t}(x, \tau)=\partial_{t} u_{0}(x, \tau), \quad(x, \tau) \in \Omega \times(-\infty, 0]
$$

onde $\Delta_{p} u$ é o operador $p$-Laplaciano, $f(u)$ é uma função contínua, possivelmente não linear, e $h(x)$ é uma força externa. Além disso, $\alpha>0$ é uma constante que será normalizada posteriormente. Como hipótese, $\mu \geq 0$ é uma função convexa decrescente. Destacamos que a função

$$
u_{0}: \Omega \times(-\infty, 0] \rightarrow \mathbb{R}
$$

é interpretada como a história de $u(t)$ para valores negativos de $t$ e é considerado um dado do problema.

Como foi mencionado na Introdução desta tese, o problema acima, sem o termo de memória, foi considerado por diversos autores e representa vibrações de placas e vigas quando a força de cisalhamento tende ao infinito. Estas observações foram apresentadas em Chueshov \& Lasiecka [10] e Yang \& Jin [48]. Em sua forma uni-dimensional, o problema também está, de certa forma, relacionado com vibrações de materiais elastoplásticos, conforme observado em Yang [47], onde é citado An \& Peirce [2].

Para considerar o problema (4.1)-(4.3) como um sistema autônomo, faremos o mesmo procedimento do Capítulo 2, introduzindo a variável $\eta$ (história do deslocamento relativo). Lembramos que tal procedimento foi desenvolvido nos trabalhos de Giorgi et al [18, 19] e Pata \& Zucchi [38], baseados em argumentos anteriores de Dafermos [14]. 
Seja

$$
\eta=\eta^{t}(x, s)=u(x, t)-u(x, t-s), \quad(x, s) \in \Omega \times \mathbb{R}^{+}, \quad t \geq 0 .
$$

Derivando formalmente (4.4) com respeito a $t$ e $s$, obtemos

$$
\eta_{t}^{t}(x, s)=-\eta_{s}^{t}(x, s)+u_{t}(x, t), \quad(x, s) \in \Omega \times \mathbb{R}^{+}, \quad t \geq 0 .
$$

Além disso, para $t=0$, obtemos a condição inicial

$$
\eta^{0}(x, s)=u_{0}(x, 0)-u_{0}(x,-s), \quad(x, s) \in \Omega \times \mathbb{R}^{+} .
$$

Assim, o termo original da memória é reescrito como

$$
\int_{0}^{\infty} \mu(s) \Delta^{2} u(t-s) d s=\left(\int_{0}^{\infty} \mu(s) d s\right) \Delta^{2} u-\int_{0}^{\infty} \mu(s) \Delta^{2} \eta^{t}(s) d s
$$

Por simplicidade, vamos considerar

$$
\alpha-\int_{0}^{\infty} \mu(s) d s=1
$$

Então, de (4.4), o problema original (4.1)-(4.3) pode ser convertido no seguinte sistema:

$$
\begin{aligned}
& u_{t t}+\Delta^{2} u-\Delta_{p} u+\int_{0}^{\infty} \mu(s) \Delta^{2} \eta^{t}(s) d s-\Delta u_{t}+f(u)=h \text { em } \Omega \times \mathbb{R}^{+}, \\
& \eta_{t}=-\eta_{s}+u_{t} \quad \text { em } \quad \Omega \times \mathbb{R}^{+} \times \mathbb{R}^{+}
\end{aligned}
$$

com condições de fronteira

$$
u=\Delta u=0 \quad \text { sobre } \quad \Gamma \times \mathbb{R}^{+}, \quad \eta=\Delta \eta=0 \quad \text { sobre } \quad \Gamma \times \mathbb{R}^{+} \times \mathbb{R}^{+},
$$

e condições iniciais

$$
u(x, 0)=u_{0}(x), \quad u_{t}(x, 0)=u_{1}(x), \quad \eta^{0}(x, s)=\eta_{0}(x, s), \quad \eta^{t}(x, 0)=0
$$

onde

$$
\left\{\begin{array}{l}
u_{0}(x)=u_{0}(x, 0), \quad x \in \Omega, \\
u_{1}(x)=\left.\partial_{t} u_{0}(x, 0)\right|_{t=0}, \quad x \in \Omega \\
\eta_{0}(x, s)=u_{0}(x, 0)-u_{0}(x,-s), \quad(x, s) \in \Omega \times \mathbb{R}^{+} .
\end{array}\right.
$$


Observação 4.1. Depois de estabelecidas as devidas hipóteses sobre o núcleo da memória $\mu$ e as definições dos espaços adequados para a história de deslocamento relativo $\eta$, segue que o problema (4.5)-(4.8) é equivalente ao problema original (4.1)-(4.3), ou seja, a partir das soluções de (4.5)-(4.8) obtemos soluções para (4.1)-(4.3) e reciprocamente. Podemos fazer isso de modo análogo ao mostrado por Grasseli \& Pata [18]. Sendo assim, por motivos de conveniência em nossas considerações futuras, vamos estudar com detalhes o problema (4.5)-(4.8).

\subsection{Resultados}

Nesta seção, apresentamos os nossos resultados e em seguida os resultados anteriores de Jorge Silva \& Ma [23, 24].

A seguir, vamos apresentar nossos dois resultados. Listamos as hipóteses (4.9)-(4.15) após os dois resultados abaixo.

Teorema 4.2. Seja $h \in L^{2}(\Omega)$. Suponhamos que as hipóteses (4.9)-(4.15) valem com as condições de sub-criticalidade

$$
p<\frac{2 N-2}{N-2} \text { se } N \geq 3 \text { e } \rho<\frac{4}{N-4} \text { se } N \geq 5 \text {. }
$$

Então, o sistema dinâmico $(\mathcal{H}, S(t))$ correspondente ao problema (4.5)-(4.8) possui um atrator exponencial generalizado $\mathcal{A}_{\exp } \subset \mathcal{H}$ com dimensão fractal finita no espaço extendido

$$
\mathcal{H}_{-1}=V_{0} \times V_{2}^{\prime} \times \mathcal{M}_{0}
$$

Além disso, por interpolação, o espaço $\mathcal{H}_{-1}$ pode ser reduzido para

$$
\mathcal{H}_{-\theta}=V_{2(1-\theta)} \times V_{2 \theta}^{\prime} \times \mathcal{M}_{2(1-\theta)},
$$

onde $0<\theta \leq 1$. 
Teorema 4.3. O atrator global correspondente ao operador de solução do problema (4.5)(4.8) (Teorema 4.6 (i) e Observação 4.7) coincide com a variedade instável $\mathbb{M}_{+}(\mathcal{N})$, onde $\mathcal{N}$ é o conjunto dos pontos estacionários de $(\mathcal{H}, S(t))$.

Agora, vamos listar as hipóteses feitas sobre o problema (4.5)-(4.8).

Para $N \in \mathbb{N}$, assumimos que:

$$
2 \leq p \leq \frac{2 N-2}{N-2} \quad \text { se } \quad N \geq 3 \quad \text { e } \quad p \geq 2 \quad \text { se } \quad N=1,2
$$

Com relação a função $f: \mathbb{R} \rightarrow \mathbb{R}$, assumimos que:

$$
f(0)=0, \quad|f(u)-f(v)| \leq k_{0}\left(1+|u|^{\rho}+|v|^{\rho}\right)|u-v|, \quad \forall u, v \in \mathbb{R}
$$

onde $k_{0}>0$ é uma constante e

$$
0<\rho \leq \frac{4}{N-4} \quad \text { se } \quad N \geq 5 \quad \text { e } \quad \rho>0 \quad \text { se } \quad 1 \leq N \leq 4
$$

Além disso, suponhamos que

$$
-k_{1} \leq \hat{f}(u) \leq f(u) u, \quad \forall u \in \mathbb{R}
$$

onde $\hat{f}(z)=\int_{0}^{z} f(s) d s$ e $k_{1} \geq 0$.

Observação 4.4. A condição (4.9) assegura que vale a seguinte cadeia de inclusões:

$$
H^{2}(\Omega) \cap H_{0}^{1}(\Omega) \hookrightarrow W_{0}^{1,2(p-1)}(\Omega) \hookrightarrow H_{0}^{1}(\Omega) \hookrightarrow L^{2}(\Omega)
$$

Analogamente, a condição (4.11) garante que

$$
H^{2}(\Omega) \cap H_{0}^{1}(\Omega) \hookrightarrow L^{2(\rho+1)}(\Omega)
$$

Então, as condições (4.10) e (4.12) incluem funções da forma

$$
f(u) \approx|u|^{\rho} u-|u|^{\alpha} u, \text { com } 0<\alpha<\rho .
$$


Com respeito ao termo de memória, assumimos que

$$
\begin{gathered}
\mu \in C^{1}\left(\mathbb{R}^{+}\right) \cap L^{1}\left(\mathbb{R}^{+}\right), \quad \int_{0}^{\infty} \mu(s) d s=\mu_{0}>0, \\
\mu(s) \geq 0, \quad \mu^{\prime}(s) \leq 0, \quad \forall s \in \mathbb{R}^{+}
\end{gathered}
$$

e que existe uma constante $k_{2}>0$ tal que

$$
\mu^{\prime}(s) \leq-k_{2} \mu(s) \leq 0, \quad \forall s \in \mathbb{R}^{+}
$$

A seguir, vamos fixar as notações que serão usadas durante todo este capítulo. Consideremos, em primeiro lugar, os espaços

$$
V_{0}=L^{2}(\Omega), \quad V_{1}=H_{0}^{1}(\Omega), \quad V_{2}=H^{2}(\Omega) \cap H_{0}^{1}(\Omega)
$$

e

$$
V_{3}=\left\{u \in H^{3}(\Omega) \mid u=\Delta u=0 \text { sobre } \Gamma\right\}
$$

equipados com os respectivos produtos internos e normas

$$
\begin{array}{rll}
(u, v)_{V_{1}}=(\nabla u, \nabla v) & \text { e } & \|u\|_{V_{1}}=\|\nabla u\|_{2}, \\
(u, v)_{V_{2}}=(\Delta u, \Delta v) & \text { e } & \|u\|_{V_{2}}=\|\Delta u\|_{2}, \\
(u, v)_{V_{3}}=(\nabla \Delta u, \nabla \Delta v) & \text { e } & \|u\|_{V_{3}}=\|\nabla \Delta u\|_{2} .
\end{array}
$$

Como usual, $(\cdot, \cdot)$ denota o produto interno em $L^{2}(\Omega)$ e $\|\cdot\|_{p}$ a norma em $L^{p}(\Omega)$. Quando não houver possibilidade de confusão, usaremos a mesma notação $\langle\cdot, \cdot\rangle$ para mencionar a dualidade entre os espaços e seus duais.

A fim de abordar a história de deslocamento relativo $\eta$ como uma nova variável, vamos considerar os seguintes espaços com peso $L_{\mu}^{2}$,

$$
\mathcal{M}_{i}:=L_{\mu}^{2}\left(\mathbb{R}^{+} ; V_{i}\right)=\left\{\xi: \mathbb{R}^{+} \rightarrow V_{i} \mid \int_{0}^{\infty} \mu(s)\|\xi(s)\|_{V_{i}}^{2} d s<\infty\right\}, \quad i=0,1,2,3,
$$

que são não vazios devido as hipóteses (4.13)-(4.14). Além disso, estes espaços são de Hilbert quando munido do produto interno e norma

$$
(\xi, \zeta)_{\mu, i}=\int_{0}^{\infty} \mu(s)(\xi(s), \zeta(s))_{V_{i}} d r, \quad\|\xi\|_{\mu, i}^{2}=\int_{0}^{\infty} \mu(s)\|\xi(s)\|_{V_{i}}^{2} d s, \quad i=0,1,2,3 .
$$


Finalmente, introduzimos os espaços de fase

$$
\mathcal{H}=V_{2} \times V_{0} \times \mathcal{M}_{2} \quad \text { e } \quad \mathcal{H}_{1}=V_{3} \times V_{1} \times \mathcal{M}_{3},
$$

equipados com as normas

$$
\|(u, v, \xi)\|_{\mathcal{H}}^{2}=\|\Delta u\|_{2}^{2}+\|v\|_{2}^{2}+\|\xi\|_{\mu, 2}^{2}
$$

e

$$
\|(u, v, \xi)\|_{\mathcal{H}_{1}}^{2}=\|\nabla \Delta u\|_{2}^{2}+\|\nabla v\|_{2}^{2}+\|\xi\|_{\mu, 3}^{2}
$$

respectivamente.

A energia do sistema sistema (4.5)-(4.8) é dado por

$$
\begin{aligned}
E(t)= & \frac{1}{2}\left\|u_{t}(t)\right\|_{2}^{2}+\frac{1}{2}\|\Delta u(t)\|_{2}^{2}+\frac{1}{p}\|\nabla u(t)\|_{p}^{p} \\
& +\frac{1}{2}\left\|\eta^{t}\right\|_{\mu, 2}^{2}+\int_{\Omega}[\hat{f}(u(t))-h u(t)] d x .
\end{aligned}
$$

A seguir, apresentamos a definição de uma solução fraca para o problema (4.5)-(4.8).

Definição 4.5. Dados $\left(u_{0}, u_{1}, \eta_{0}\right) \in \mathcal{H}$, diremos que uma função $z=\left(u, u_{t}, \eta\right) \in$ $C([0, T], \mathcal{H})$ é uma solução fraca para o problema (4.5)-(4.8) se $z(0)=\left(u_{0}, u_{1}, \eta_{0}\right)$ e

$$
\begin{aligned}
& \frac{d}{d t}\left(u_{t}, \omega\right)+(\Delta u, \Delta \omega)+\left\langle|\nabla u|^{p-2} \nabla u, \nabla \omega\right\rangle \\
& \quad+\int_{0}^{\infty} \mu(s)(\Delta \eta(s), \Delta \omega) d s+\left(\nabla u_{t}, \nabla \omega\right)+(f(u), \omega)=(h, \omega), \\
& \left(\partial_{t} \eta+\partial_{s} \eta, \xi\right)_{\mu, 2}=\left(u_{t}, \xi\right)_{\mu, 2},
\end{aligned}
$$

quase sempre em $[0, T]$, para todo $\omega \in V_{2}$ e $\xi \in \mathcal{M}_{2}$.

Os dois próximos resultados foram provados em Jorge Silva \& Ma [23, 24].

Teorema 4.6. Seja $h \in L^{2}(\Omega)$. Sob as hipóteses (4.9)-(4.15), temos:

(i) Se $\left(u_{0}, u_{1}, \eta_{0}\right) \in \mathcal{H}$, então o problema (4.5)-(4.8) possui uma solução fraca

$$
\left(u, u_{t}, \eta\right) \in C([0, T], \mathcal{H}), \quad \forall T>0
$$


satisfazendo

$u \in L^{\infty}\left(0, T ; V_{2}\right), \quad u_{t} \in L^{\infty}\left(0, T ; V_{0}\right) \cap L^{2}\left(0, T ; V_{1}\right), \quad \eta \in L^{\infty}\left(0, T ; \mathcal{M}_{2}\right)$.

(ii) Se $\left(u_{0}, u_{1}, \eta_{0}\right) \in \mathcal{H}_{1}$, então a solução fraca do problema (4.5)-(4.8) possui maior regularidade, a saber,

$u \in L^{\infty}\left(0, T ; V_{3}\right), \quad u_{t} \in L^{\infty}\left(0, T ; V_{1}\right) \cap L^{2}\left(0, T ; V_{2}\right), \quad \eta \in L^{\infty}\left(0, T ; \mathcal{M}_{3}\right)$.

(iii) Em ambos os casos, a solução $\left(u, u_{t}, \eta\right)$ depende continuamente dos dados iniciais em H. Mais precisamente, sejam $z_{i}=\left(u^{i}, u_{t}^{i}, \eta^{i}\right)$ duas soluções do problema (4.5)-(4.8) correspondentes aos dados iniciais $z_{i, 0}=\left(u_{0}^{i}, u_{1}^{i}, \eta_{0}^{i}\right)$, para $i=1,2$. Então, vale a seguinte estimativa:

$$
\left\|z_{1}(t)-z_{2}(t)\right\|_{\mathcal{H}} \leq e^{c t}\left\|z_{1,0}-z_{2,0}\right\|_{\mathcal{H}}, \quad t \in[0, T]
$$

para alguma constante $c>0$. Em particular, o problema (4.5)-(4.8) possui uma única solução fraca.

(iv) Se B é limitado em $\mathcal{H}$, então as soluções $z(t)=\left(u(t), u_{t}(t), \eta^{t}\right)$ com dados iniciais em $B$ satisfazem a estimativa

$$
\|z(t)\|_{\mathcal{H}} \leq C_{B}, \quad \forall t \geq 0
$$

onde $C_{B}>0$ é uma constante dependente de B, mas não de $t$.

Observação 4.7. Utilizando um argumento análogo ao observado no início do Capítulo 3, o resultado de boa colocação do problema, dado pelo Teorema 4.5, garante que o operador solução $S(t) z_{0}=\left(u(t), u_{t}(t), \eta^{t}\right)$ é um semigrupo fortemente contínuo no espaço $\mathcal{H}=V_{2} \times V_{1} \times \mathcal{M}_{2}$. Ou seja, o problema (4.5)-(4.8) corresponde a um sistema dinâmico $(\mathcal{H}, S(t))$.

Teorema 4.8. Sob as hipóteses do Teorema 4.6 temos: 
(i) O sistema dinâmico $(\mathcal{H}, S(t))$ correspondente ao problema (4.5)-(4.8) possui um atrator global compacto e conexo.

(ii) Sob as condições de sub-criticalidade

$$
p<\frac{2 N-2}{N-2} \text { se } N \geq 3 \text { e } \rho<\frac{4}{N-4} \text { se } N \geq 5,
$$

o atrator correspondente possui dimensão fractal finita.

\subsection{Existência de atratores exponenciais}

A prova do Teorema 4.2 é baseada no conceito de quasi-estabilidade (ver Definição 1.24). Jorge Silva \& Ma [23] mostraram a propriedade de quasi-estabilidade de $(\mathcal{H}, S(t))$ para subconjuntos limitados de $\mathcal{H}$, que é baseada numa certa desigualdade de estabilização. Para conveniência do leitor vamos repetir a prova desse resultado.

Lema 4.9 (Desigualdade de estabilização). Sob as hipóteses do Teorema 4.6, dado um conjunto limitado $B \subset \mathcal{H}$, sejam $z_{i}=\left(u^{i}, u_{t}^{i}, \eta^{i}\right)$ duas soluções fracas do problema (4.5)(4.8) tais que $z_{i}(0)=\left(u_{0}^{i}, u_{1}^{i}, \eta_{0}^{i}\right) \in B$, para $i=1,2$. Então, vale a seguinte estimativa

$$
\begin{aligned}
\| z_{1}(t) & -z_{2}(t)\left\|_{\mathcal{H}}^{2} \leq 3 e^{-\nu t}\right\| z_{1}(0)-z_{2}(0) \|_{\mathcal{H}}^{2} \\
& +K_{B} \int_{0}^{t} e^{-\nu(t-\tau)}\left(\left\|\nabla\left(u^{1}(\tau)-u^{2}(\tau)\right)\right\|_{2(p-1)}^{2}+\left\|u^{1}(\tau)-u^{2}(\tau)\right\|_{2(\rho+1)}^{2}\right) d \tau
\end{aligned}
$$

para todo $t>0$, onde $\nu>0$ é uma constante pequena e $K_{B}>0$ é uma constante dependendo do tamanho de $B$.

Demonstração. Seja $B \subset \mathcal{H}$ limitado. Dadas duas soluções $z_{i}=\left(u^{i}, u_{t}^{i}, \eta^{i}\right)$ de (4.5)-(4.8) $\operatorname{com} z_{i}(0)=\left(u_{0}^{i}, u_{1}^{i}, \eta_{0}^{i}\right) \in B, i=1,2$, então a função $\left(w, w_{t}, \zeta\right)=z_{1}-z_{2}$ é solução (no sentido do Teorema 4.6) do problema

$$
\begin{gathered}
w_{t t}+\Delta^{2} w-\Delta_{p} u^{1}+\Delta_{p} u^{2}+\int_{0}^{\infty} \mu(s) \Delta^{2} \zeta^{t}(s) d s-\Delta w_{t}+f\left(u^{1}\right)-f\left(u^{2}\right)=0, \\
\zeta_{t}=-\zeta_{s}+w_{t}
\end{gathered}
$$


com condição inicial

$$
\left(w(0), w_{t}(0), \zeta^{0}\right)=z_{1}(0)-z_{2}(0)
$$

Analogamente como na prova de dependência contínua de soluções, segue que

$$
\begin{aligned}
\frac{1}{2} \frac{d}{d t} & \left\{\|\Delta w(t)\|_{2}^{2}+\left\|w_{t}(t)\right\|_{2}^{2}+\left\|\zeta^{t}\right\|_{\mu, 2}^{2}\right\}+\left\|\nabla w_{t}(t)\right\|_{2}^{2} \\
& =\left\langle\Delta_{p} u^{1}(t)-\Delta_{p} u^{2}(t), w_{t}(t)\right\rangle-\left(f\left(u^{1}(t)\right)-f\left(u^{2}(t)\right), w_{t}(t)\right)-\left(\zeta^{t}, \zeta_{s}^{t}\right)_{\mu, 2}
\end{aligned}
$$

Primeiramente, notemos que

$$
-\left(\zeta^{t}, \zeta_{s}^{t}\right)_{\mu, 2} \leq-\frac{k_{2}}{2} \int_{0}^{\infty} \mu(s)\left\|\Delta \zeta^{t}(s)\right\|_{2}^{2} d s=-\frac{k_{2}}{2}\left\|\zeta^{t}\right\|_{\mu, 2}^{2}
$$

No que segue, vamos usar o mesmo parâmetro $C_{B}$ para denotar diferentes constantes positivas que dependem do conjunto limitado $B$, mas não de $t>0$.

Usando a desigualdade de Hölder generalizada com $\frac{p-2}{2(p-1)}+\frac{1}{2(p-1)}+\frac{1}{2}=1$ e a condição (4.9) temos

$$
\begin{aligned}
\mid\left\langle\Delta_{p} u^{1}(t)-\right. & \left.\Delta_{p} u^{2}(t), w_{t}(t)\right\rangle \mid \\
& \leq\left.\int_{\Omega}|| \nabla u^{1}(t)\right|^{p-2} \nabla u^{1}(t)-\left|\nabla u^{2}(t)\right|^{p-2} \nabla u^{2}(t)|| \nabla w_{t}(t) \mid d x \\
& \leq M_{p} \int_{\Omega}\left(\left|\nabla u^{1}(t)\right|^{p-2}+\left|\nabla u^{2}(t)\right|^{p-2}\right)|\nabla w(t)|\left|\nabla w_{t}(t)\right| d x \\
& \leq M_{p}\left(\left\|\nabla u^{1}(t)\right\|_{2(p-1)}^{p-2}+\left\|\nabla u^{2}(t)\right\|_{2(p-1)}^{p-2}\right)\|\nabla w(t)\|_{2(p-1)}\left\|\nabla w_{t}(t)\right\|_{2} \\
& \leq C_{B}\|\nabla w(t)\|_{2(p-1)}\left\|\nabla w_{t}(t)\right\|_{2} .
\end{aligned}
$$

Logo, pela desigualdade de Young existe uma constante $C_{B}>0$ tal que

$$
\left|\left\langle\Delta_{p} u^{1}(t)-\Delta_{p} u^{2}(t), w_{t}(t)\right\rangle\right| \leq \frac{C_{B}}{2}\|\nabla w(t)\|_{2(p-1)}^{2}+\frac{1}{4}\left\|\nabla w_{t}(t)\right\|_{2}^{2}
$$

Além disso, como $\frac{\rho}{2(\rho+1)}+\frac{1}{2(\rho+1)}+\frac{1}{2}=1$, então, novamente pela desigualdade de Hölder generalizada, das condições (4.10)-(4.11), da estimativa (4.22) e pela desigualdade 
de Poincaré, obtemos

$$
\begin{aligned}
\mid\left(f\left(u^{1}(t)\right)\right. & \left.-f\left(u^{2}(t)\right), w_{t}(t)\right) \mid \\
& \leq k_{0} \int_{\Omega}\left(1+\left|u^{1}(t)\right|^{\rho}+\left|u^{2}(t)\right|^{\rho}\right)|w(t)|\left|w_{t}(t)\right| d x \\
& \leq k_{0}\left(|\Omega|^{\frac{\rho}{2(\rho+1)}}+\left\|u^{1}(t)\right\|_{2(\rho+1)}^{\rho}+\left\|u^{2}(t)\right\|_{2(\rho+1)}^{\rho}\right)\|w(t)\|_{2(\rho+1)}\left\|w_{t}(t)\right\|_{2} \\
& \leq C_{B}\|w(t)\|_{2(\rho+1)}\left\|\nabla w_{t}(t)\right\|_{2} .
\end{aligned}
$$

Novamente pela desigualdade de Young, existe uma constante $C_{B}>0$ tal que

$$
\left|\left(f\left(u^{1}(t)\right)-f\left(u^{2}(t)\right), w_{t}(t)\right)\right| \leq \frac{C_{B}}{2}\|w(t)\|_{2(\rho+1)}^{2}+\frac{1}{4}\left\|\nabla w_{t}(t)\right\|_{2}^{2} .
$$

Substituindo (4.28)-(4.30) em (4.27), então

$$
\frac{d}{d t} F(t) \leq-\left\|\nabla w_{t}(t)\right\|_{2}^{2}-k_{2}\left\|\zeta^{t}\right\|_{\mu, 2}^{2}+C_{B}\left(\|\nabla w(t)\|_{2(p-1)}^{2}+\|w(t)\|_{2(\rho+1)}^{2}\right)
$$

onde denotamos

$$
F(t)=\|\Delta w(t)\|_{2}^{2}+\left\|w_{t}(t)\right\|_{2}^{2}+\left\|\zeta^{t}\right\|_{\mu, 2}^{2}:=\left\|z_{1}(t)-z_{2}(t)\right\|_{\mathcal{H}}^{2}, \quad t \geq 0 .
$$

Agora, consideremos a perturbação

$$
F_{\varepsilon}(t)=F(t)+\varepsilon \Phi(t), \quad \varepsilon>0
$$

onde $\varepsilon>0$ será fixado posteriormente e

$$
\Phi(t)=\int_{\Omega} w_{t}(t) w(t) d x
$$

Afirmação 1: Existe uma constante $C_{1}>0$ tal que

$$
\left|F_{\varepsilon}(t)-F(t)\right| \leq \varepsilon C_{1} F(t), \quad \forall t \geq 0, \forall \varepsilon>0
$$

Prova. Segue diretamente da definição de $F$. 
Afirmação 2: Existem constantes $\varepsilon_{1}>0 e C_{B}>0$ tais que

$$
\frac{d}{d t} F_{\varepsilon}(t) \leq-\frac{\varepsilon}{2} F(t)+C_{B}\left(\|\nabla w(t)\|_{2(p-1)}^{2}+\|w(t)\|_{2(\rho+1)}^{2}\right),
$$

para todo $t \geq 0, e \varepsilon \in\left(0, \varepsilon_{1}\right]$.

Prova. É suficiente provarmos que existem constantes $C_{2}, C_{3}>0$ e uma constante $C_{B}>0$, dependendo do conjunto limitado $B$, tais que

$$
\begin{aligned}
\frac{d}{d t} \Phi(t) \leq & -\frac{1}{2} F(t)+C_{2}\left\|\nabla w_{t}(t)\right\|_{2}^{2}+C_{3}\left\|\zeta^{t}\right\|_{\mu, 2}^{2} \\
& +C_{B}\left(\|\nabla w(t)\|_{2(p-1)}^{2}+\|w(t)\|_{2(\rho+1)}^{2}\right) .
\end{aligned}
$$

De fato, combinando esta estimativa com (4.31), escolhendo $\varepsilon_{1}=\min \left\{\frac{1}{C_{2}}, \frac{k_{2}}{C_{3}}\right\}>0$ e tomando $\varepsilon \in\left(0, \varepsilon_{1}\right]$, então

$$
\begin{aligned}
\frac{d}{d t} F_{\varepsilon}(t)= & \frac{d}{d t} F(t)+\varepsilon \frac{d}{d t} \Phi(t) \\
\leq & -\frac{\varepsilon}{2} F(t)-\left(1-\varepsilon C_{2}\right)\left\|\nabla w_{t}(t)\right\|_{2}^{2}-\left(k_{2}-\varepsilon C_{3}\right)\left\|\zeta^{t}\right\|_{\mu, 2}^{2} \\
& +(1+\varepsilon) C_{B}\left(\|\nabla w(t)\|_{2(p-1)}^{2}+\|w(t)\|_{2(\rho+1)}^{2}\right) \\
\leq & -\frac{\varepsilon}{2} F(t)+\left(1+\varepsilon_{1}\right) C_{B}\left(\|\nabla w(t)\|_{2(p-1)}^{2}+\|w(t)\|_{2(\rho+1)}^{2}\right) .
\end{aligned}
$$

Isto mostra (4.35) para alguma constante $C_{B}>0$ (no lugar de $\left(1+\varepsilon_{1}\right) C_{B}$ ).

No que segue, vamos mostrar a desigualdade (4.36). Com efeito, derivando a função em (4.33), usando a equação (4.24) no sentido fraco, adicionando e subtraindo $\frac{1}{2} F(t)$ na expressão resultante, obtemos

$$
\frac{d}{d t} \Phi(t)=-\frac{1}{2} F(t)+\frac{3}{2}\left\|w_{t}(t)\right\|_{2}^{2}-\frac{1}{2}\|\Delta w(t)\|_{2}^{2}+\frac{1}{2}\left\|\zeta^{t}\right\|_{\mu, 2}^{2}+\sum_{j=1}^{4} L_{j},
$$

onde

$$
\begin{aligned}
& L_{1}=-\int_{0}^{\infty} \mu(s)\left(\Delta \zeta^{t}(s), \Delta w(t)\right) d s, \\
& L_{2}=-\int_{\Omega} \nabla w_{t}(t) \cdot \nabla w(t) d x \\
& L_{3}=\left\langle\Delta_{p} u^{1}(t)-\Delta_{p} u^{2}(t), w(t)\right\rangle, \\
& L_{4}=-\left(f\left(u^{1}(t)\right)-f\left(u^{2}(t)\right), w(t)\right) .
\end{aligned}
$$


Agora, vamos estimar os termos $L_{1}, L_{2}, L_{3}$ e $L_{4}$. Temos:

$$
\begin{gathered}
\left|L_{1}\right| \leq \sigma \mu_{0}\|\Delta w(t)\|_{2}^{2}+\frac{1}{4 \sigma}\left\|\zeta^{t}\right\|_{\mu, 2}^{2}, \\
\left|L_{2}\right| \leq \sigma d_{1}\|\Delta w(t)\|_{2}^{2}+\frac{1}{4 \sigma}\left\|\nabla w_{t}(t)\right\|_{2}^{2},
\end{gathered}
$$

onde $d_{1}>0$ é a constante da imersão $V_{2} \hookrightarrow V_{1}$.

Em segundo lugar, utilizando os mesmos argumentos para obter (4.29)-(4.30), e usando $w$ no lugar de $w_{t}$, obtemos mais as seguintes estimativas:

$$
\left|L_{3}\right| \leq C_{B}\|\nabla w(t)\|_{2(p-1)}^{2}
$$

$\mathrm{e}$

$$
\left|L_{4}\right| \leq C_{B}\|w(t)\|_{2(\rho+1)}^{2}
$$

para alguma constante $C_{B}>0$ dependendo do conjunto absorvente limitado $B$.

Retornando a (4.37) e substituindo estas quatro últimas estimativas, chegamos a

$$
\begin{aligned}
\frac{d}{d t} \Phi(t) \leq & -\frac{1}{2} F(t)+\left(\frac{3}{2} d_{2}+\frac{1}{4 \sigma}\right)\left\|\nabla w_{t}(t)\right\|_{2}^{2}+\left(\frac{1}{2}+\frac{1}{4 \sigma}\right)\left\|\zeta^{t}\right\|_{\mu, 2}^{2} \\
& +C_{B}\left(\|\nabla w(t)\|_{2(p-1)}^{2}+\|w(t)\|_{2(\rho+1)}^{2}\right)-\left(\frac{1}{2}-\sigma\left(\mu_{0}+d_{1}\right)\right)\|\Delta w(t)\|_{2}^{2},
\end{aligned}
$$

onde $d_{2}>0$ é a constante de imersão $V_{1} \hookrightarrow V_{0}$. Portanto, tomando

$$
\sigma \leq \frac{1}{2\left(\mu_{0}+2 d_{1}\right)}, \quad C_{2}=\frac{3}{2} d_{2}+\frac{1}{4 \sigma} \quad \text { e } \quad C_{3}=\frac{1}{2}+\frac{1}{4 \sigma},
$$

a desigualdade (4.36) fica provada. Isto termina a prova da Afirmação 2.

Agora, podemos concluir a prova da desigualdade de estabilização (4.23) como segue. Definamos $\varepsilon_{0}=\min \left\{\frac{1}{2 C_{1}}, \varepsilon_{1}\right\}$ e consideremos $\varepsilon \leq \varepsilon_{0}$. Assim, estimativa (4.34) implica que

$$
\frac{1}{2} F(t) \leq F_{\varepsilon}(t) \leq \frac{3}{2} F(t), \quad t \geq 0
$$

Combinando, de forma padrão, a desigualdade (4.35) e a segunda desigualdade em (4.38), resulta que

$$
F_{\varepsilon}(t) \leq e^{-\varepsilon t / 3} F_{\varepsilon}(0)+C_{B} \int_{0}^{t} e^{-\varepsilon(t-\tau) / 3}\left(\|\nabla w(\tau)\|_{2(p-1)}^{2}+\|w(\tau)\|_{2(\rho+1)}^{2}\right) d \tau, \quad t \geq 0 .
$$


Usando novamente (4.38), concluímos que

$$
F(t) \leq 3 e^{-\nu t} F(0)+K_{B} \int_{0}^{t} e^{-\nu(t-\tau)}\left(\|\nabla w(\tau)\|_{2(p-1)}^{2}+\|w(\tau)\|_{2(\rho+1)}^{2}\right) d \tau, \quad t \geq 0
$$

onde $\nu=\varepsilon / 3>0$ é uma constante positiva pequena e $K_{B}=2 C_{B}>0$ é uma constante dependendo de $B$. Mantendo em mente a identidade (4.32) e que $w=u^{1}-u^{2}$, então a estimativa (4.39) implica na desigualdade de estabilidade (4.23), o que encerra a prova da Lemma 4.9.

Lema 4.10 (Quasi-estabilidade). Sob as hipóteses do Teorema 4.2, o sistema dinâmico $(\mathcal{H}, S(t))$ correspondente é quasi-estável em qualquer subconjunto limitado e positivamente invariante $B \subset \mathcal{H}$.

Demonstração. Seja $X=V_{2}, Y=V_{0}$ e $Z=\mathcal{M}_{2}$. Então, como $S(t)$ é o operador solução do problema (4.5)-(4.8), então as condições (1.3) e (1.4) estão verificadas.

Assim, o resultado segue da desigualdade de estabilização (Lema 4.9). Os detalhes estão provados em [23].

Observação 4.11. M. A. Jorge Silva \& T. F. Ma [23] mostraram que o sistema dinâmico $(\mathcal{H}, S(t))$ correspondente ao problema (4.5)-(4.8) é dissipativo. Assim, seja $\mathcal{B} \subset \mathcal{H}$ um conjunto absorvente limitado para $(\mathcal{H}, S(t))$. Então, existe $T_{B}$ tal que

$$
S(t) \mathcal{B} \subset \mathcal{B}, \quad \forall t \geq T_{B}
$$

Com isto concluímos que o conjunto

$$
\mathcal{B}_{0}:=\bigcup_{t \geq T_{B}} S(t) \mathcal{B}
$$

é absorvente, limitado, positivamente invariante e $\mathcal{B}_{0} \subset \mathcal{B}$. Logo, pelo Lema 4.10, $(\mathcal{H}, S(t))$ é quasi-estável em $\mathcal{B}_{0}$. 
Prova do Teorema 4.2. Vamos mostrar que $t \mapsto S(t) z$ é Hölder continua em $\mathcal{H}_{-1}$ para todo $z \in \mathcal{B}_{0}$. Seja $z=\left(u_{0}, u_{1}, \eta^{0}\right) \in \mathcal{B}_{0}$. De (4.19) e das equações (4.5)-(4.6) segue que

$$
\left(u_{t}, u_{t t}, \eta_{t}\right) \in L^{\infty}\left(0, T ; V_{0}\right) \times L^{2}\left(0, T ; V_{2}^{\prime}\right) \times L^{\infty}\left(0, T ; \mathcal{M}_{0}\right), \quad \forall T>0
$$

Logo,

$$
\left\|\frac{d}{d t} z(t)\right\|_{\mathcal{H}_{-1}} \leq C_{B_{0}, T},
$$

onde $z(t)=\left(u(t), u_{t}(t), \eta^{t}\right)$. Escrevendo $\left(u(t), u_{t}(t), \eta^{t}\right)=S(t) z:=z(t)$ obtemos:

$$
\begin{aligned}
\left\|S\left(t_{2}\right) z-S\left(t_{1}\right) z\right\|_{\mathcal{H}_{-1}} & =\left\|\int_{t_{1}}^{t_{2}} \frac{d}{d s} z(s) d s\right\|_{\mathcal{H}_{-1}} \\
& \leq \int_{t_{1}}^{t_{2}}\left\|\frac{d}{d s} z(s)\right\|_{\mathcal{H}_{-1}} d s \\
& \leq\left(\int_{0}^{T}\left\|\left(u_{t}(s), u_{t t}(s), \eta_{t}^{s}\right)\right\|_{\mathcal{H}_{-1}}^{2} d s\right)^{1 / 2}\left|t_{2}-t_{1}\right|^{1 / 2} \\
& \leq C_{\mathcal{B}_{0}, T}\left|t_{2}-t_{1}\right|^{1 / 2}
\end{aligned}
$$

para todo $t_{1}, t_{2} \in[0, T]$. Do Teorema 1.27, segue que o sistema dinâmico $(\mathcal{H}, S(t))$ possui um atrator exponencial fractal $\mathcal{A}_{\exp }$ com dimensão fractal finita em $\mathcal{H}_{-1}$.

Vamos agora construir o conjunto $\mathcal{H}_{-\theta}:=V_{2(1-\theta)} \times V_{2 \theta}^{\prime} \times \mathcal{M}_{2(1-\theta)}$. Sabemos que

$$
V_{2} \hookrightarrow \hookrightarrow V_{0}, \quad V_{0}^{\prime} \hookrightarrow \hookrightarrow V_{2}^{\prime} \quad \text { e } \quad \mathcal{M}_{2} \hookrightarrow \hookrightarrow \mathcal{M}_{0}
$$

Da teoria de interpolação, podemos construir $V_{2(1-\theta)}, V_{2 \theta}^{\prime}$ e $\mathcal{M}_{2(1-\theta)} \operatorname{com} \theta \in(0,1]$ tais que

$$
\begin{aligned}
& \|u\|_{V_{2(1-\theta)}} \leq c_{1}(\theta)\|u\|_{V_{2}}^{1-\theta}\|u\|_{V_{0}}^{\theta}, \quad u \in V_{2} \\
& \|v\|_{V_{2 \theta}^{\prime}} \leq c_{2}(\theta)\|v\|_{V_{0}^{\prime}}^{1-\theta}\|v\|_{V_{2}^{\prime}}^{\theta}, \quad v \in V_{0}^{\prime} \\
& \|\xi\|_{\mathcal{M}_{2(1-\theta)}} \leq c_{3}(\theta)\|\xi\|_{\mathcal{M}_{2}}^{1-\theta}\|\xi\|_{\mathcal{M}_{0}}^{\theta}, \quad \xi \in \mathcal{M}_{2}
\end{aligned}
$$

Assim,

$$
\begin{aligned}
\|u\|_{V_{2(1-\theta)}}^{2}+\|v\|_{V_{2 \theta}^{\prime}}^{2}+\|\xi\|_{\mathcal{M}_{2(1-\theta)}}^{2} \leq & c_{1}(\theta)^{2}\left(\|u\|_{V_{2}}^{2}\right)^{1-\theta}\left(\|u\|_{V_{0}}^{2}\right)^{\theta} \\
& +c_{2}(\theta)^{2}\left(\|v\|_{V_{0}^{\prime}}^{2}\right)^{1-\theta}\left(\|v\|_{V_{2}^{\prime}}^{2}\right)^{\theta} \\
& +c_{3}(\theta)^{2}\left(\|\xi\|_{\mathcal{M}_{2}}^{2}\right)^{1-\theta}\left(\|\xi\|_{\mathcal{M}_{0}}^{2}\right)^{\theta}
\end{aligned}
$$


Então, para $C(\theta)^{2}=\max \left\{c_{1}(\theta)^{2}, c_{2}(\theta)^{2}, c_{3}(\theta)^{2}\right\}$, temos

$$
\begin{aligned}
& \|u\|_{V_{2(1-\theta)}}^{2}+\|v\|_{V_{2 \theta}^{\prime}}^{2}+\|\xi\|_{\mathcal{M}_{2(1-\theta)}}^{2} \\
& \leq C(\theta)^{2}\left(\|u\|_{V_{2}}^{2}+\|v\|_{V_{0}^{\prime}}^{2}+\|\xi\|_{\mathcal{M}_{2}}^{2}\right)^{1-\theta}\left(\|u\|_{V_{0}}^{2}+\|v\|_{V_{2}^{\prime}}^{2}+\|\xi\|_{\mathcal{M}_{0}}^{2}\right)^{\theta}
\end{aligned}
$$

Logo,

$$
\|(u, v, \xi)\|_{\mathcal{H}_{-\theta}}^{2} \leq C(\theta)^{2}\left(\|(u, v, \xi)\|_{\mathcal{H}^{2}}^{2}\right)^{1-\theta}\left(\|(u, v, \xi)\|_{\mathcal{H}_{-1}}^{2}\right)^{\theta}
$$

ou seja,

$$
\|(u, v, \xi)\|_{\mathcal{H}_{-\theta}} \leq C(\theta)\|(u, v, \xi)\|_{\mathcal{H}}^{1-\theta}\|(u, v, \xi)\|_{\mathcal{H}_{-1}}^{\theta}
$$

para todo $(u, v, \xi) \in \mathcal{H}$ e para todo $\theta \in(0,1]$. Assim, basta mostrarmos que a função $t \mapsto S(t) z$ é Hölder contínua em $\mathcal{H}_{-\theta}:=V_{2(1-\theta)} \times V_{2 \theta}^{\prime} \times \mathcal{M}_{2(1-\theta)}$, onde $\theta \in(0,1]$ e $z \in \mathcal{B}_{0}$. Usando a limitação de $z(t)$ em (4.22) e as desigualdades (4.40) e (4.41) obtemos:

$$
\begin{aligned}
\left\|S\left(t_{2}\right) z-S\left(t_{1}\right) z\right\|_{\mathcal{H}_{-\theta}} & \leq C(\theta)\left\|S\left(t_{2}\right) z-S\left(t_{1}\right) z\right\|_{\mathcal{H}}^{1-\theta}\left\|S\left(t_{2}\right) z-S\left(t_{1}\right) z\right\|_{\mathcal{H}_{-1}}^{\theta} \\
& \leq C_{\theta, T}\left\|S\left(t_{2}\right) z-S\left(t_{1}\right) z\right\|_{\mathcal{H}_{-1}}^{\theta} \\
& \leq C_{\mathcal{B}_{0}, \theta, T}\left|t_{2}-t_{1}\right|^{\theta / 2}
\end{aligned}
$$

para todo $t_{1}, t_{2} \in[0, T]$ e $\theta \in(0,1]$.

Isto encerra a prova Teorema 4.2.

\subsection{Caracterização do atrator global}

Nesta seção, provamos o Teorema 4.3.

Lema 4.12. Nas hipóteses do Teorema 4.8, o sistema dinâmico correspondente é gradiente.

Demonstração. Consideremos o funcional de Lyapunov $\Psi(S(t) z)=E(t)$, onde $E(t)$ é a energia do sistema. Então,

$$
\frac{d}{d t} \Psi(u(t))=-\left\|\nabla u_{t}(t)\right\|_{2}^{2}+\frac{1}{2} \int_{0}^{\infty} \mu^{\prime}(s)\left\|\Delta \eta^{t}(s)\right\|_{2}^{2} d s \leq 0 .
$$


Portanto, o funcional de Lyapunov é decrescente. Suponhamos agora que, para uma trajetória $z(t)=\left(u(t), u_{t}(t), \eta^{t}\right)$, temos $\Psi(z(t))=z(t)$ para todo $t \geq 0$. Logo,

$$
\frac{d}{d t} \Psi(z(t))=0, \quad \forall t \geq 0
$$

ou seja,

$$
\left\|\nabla u_{t}(t)\right\|_{2}^{2}+\frac{1}{2} \int_{0}^{\infty}-\mu^{\prime}(s)\left\|\Delta \eta^{t}(s)\right\|_{2}^{2} d s=0, \quad \forall t \geq 0 .
$$

Como ambos termos do lado esquerdo possuem o mesmo sinal, concluímos que

$$
\left\|\nabla u_{t}(t)\right\|_{2}^{2}=0 \quad \text { e } \quad \int_{0}^{\infty} \mu^{\prime}(s)\left\|\Delta \eta^{t}(s)\right\|_{2}^{2} d s=0, \quad \forall t \geq 0
$$

Assim, $u_{t}(t)=0$ e $\eta^{t}=0$, para todo $t$. Assim, concluímos que $z(t)=(u, 0,0)$, o que mostra que $z(t)$ é uma solução estacionária do problema (4.5)-(4.8). Conforme a Definição 1.29, mostramos que $\Psi(t)$ é um funcional de Lyapunov estrito sobre $\mathcal{H}$. Portanto, o sistema em questão é gradiente.

Prova do Teorema 4.3: Como foi mostrado que o sistema $(\mathcal{H}, S(t))$ é gradiente, segue do Teorema 1.30 que, o único atrator global $\mathcal{A}$ de $(\mathcal{H}, S(t))$ é caracterizado por

$$
\mathcal{A}=\mathbb{M}_{+}(\mathcal{N})
$$

onde $\mathcal{N}$ é o conjunto dos pontos estacionários de $(\mathcal{H}, S(t))$. 



\section{Referências Bibliográficas}

[1] R. A. Adams \& J. J. F. Fournier, Sobolev Spaces, second edition, Pure and Applied Mathematics (Amsterdam), 140. Elsevier/Academic Press, Amsterdam, 2003.

[2] L. An \& A. Peirce, A weakly nonlinear analysis of elastoplastic-microstructure models, SIAM J. Appl. Math. 55 (1995) 136-155.

[3] D. Andrade, M. A. Jorge Silva \& T. F. Ma, Exponential stability for a plate equation with p-Laplacian and memory terms, Math. Meth. Appl. Sci. 35 (2012) 417-426.

[4] Rawlilson O. Araújo, T. F. Ma \& Yuming Qin, Long-time behavior of a quasilinear viscoelastic equation with past history, J. Differential Equations 254 (2013) 4066-4087.

[5] A. V. Babin \& M. I. Vishik, Attractors of Evolution Equations, Studies in Mathematics and its Applications 25, North-Holland, Amsterdam, 1992.

[6] A. Benaissa \& A. Guesmia, Energy decay for the wave equations of $\phi$-Laplacian type with weakly nonlinear dissipation, Electron. J. Differential Equations (2008), no. 109, $1-22$.

[7] H. Brézis, Analyse Fonctionnelle: Théorie et Applications, Paris, Masson, 1983. 
[8] M. M. Cavalcanti, V. N. Domingos Cavalcanti \& J. Ferreira, Existence and uniform decay for a non-linear viscoelastic equation with strong damping, Math. Meth. Appl. Sci. 24 (2001) 1043-1053.

[9] M. M. Cavalcanti \& H. Portillo Oquendo, Frictional versus viscoelastic damping in a semilinear wave equation, SIAM J. Control Optim. 42 (2003) 1310-1324.

[10] I. Chueshov \& I. Lasiecka, Existence, uniqueness of weak solutions and global attractors for a class of nonlinear 2D Kirchhoff-Boussinesq models, Discrete Contin. Dyn. Syst. 15 (2006) 777-809.

[11] I. Chueshov \& I. Lasiecka, Long-Time Behavior of Second Order Evolution Equations with Nonlinear Damping, Mem. Amer. Math. Soc. 195, no. 912, Providence, 2008.

[12] I. Chueshov \& I. Lasiecka, On global attractor for 2D Kirchhoff-Boussinesq model with supercritical nonlinearity, Comm. Partial Differential Equations 36 (2011) 67-99.

[13] I. Chueshov \& I. Lasiecka, Von Karman Evolution Equations: Well-Posedness and Long-Time Dynamics, Springer Monographs in Mathematics, Springer, New York, 2010.

[14] C. M. Dafermos Asymptotic stability in viscoelasticity, Arch. Rational Mech. Anal. 37 (1970) 297-308.

[15] A. Eden, C. Foias, B. Nicolaenko \& R. Temam, Exponential attractors for dissipative evolution equations, Research in Applied Mathematics, vol. 37, John Wiley \& Sons, New York, 1994.

[16] L. C. Evans, Partial Differential Equations, Graduate Studies in Mathematics, vol. 19, American Mathematical Society, Providence, 1998.

[17] M. Fabrizio \& A. Morro, Mathematical Problems in Linear Viscoelasticity, SIAM Studies in Applied Mathematics 12, SIAM, Philadelphia, 1992. 
[18] C. Giorgi, M. Grasseli \& V. Pata, Well-posedness and longtime behavior of the phasefield model with memory in a history space setting, Quart. Appl. Math. 59 (2001) 701736.

[19] C. Giorgi, J. E. Muñoz Rivera \& V. Pata, Global attractors for a semilinear hyperbolic equation in viscoelasticity, J. Math. Anal. Appl. 260 (2001) 83-99.

[20] J. K. Hale, Asymptotic Behavior of Dissipative Systems, Mathematical Surveys and Monographs, 25, American Mathematical Society, Providence, 1988.

[21] X. Han \& M. Wang, General decay of energy for a viscoelastic equation with nonlinear damping, Math. Meth. Appl. Sci. 32 (2009) 346-358.

[22] X. Han \& M. Wang, Global existence and uniform decay for a nonlinear viscoelastic equation with damping, Nonlinear Anal. 70 (2009) 3090-3098.

[23] M. A. Jorge Silva \& T. F. Ma, Long-time dynamics for a class of Kirchhoff models with memory, J. Math. Phys. 54, article 021505 (2013).

[24] M. A. Jorge Silva \& T. F. Ma, On a viscoelastic plate equation with history setting and perturbation of p-Laplacian type, IMA J. Appl. Math. (a aparecer em 2013).

[25] E. Kreyszig, Introductory Functional Analysis with Applications, John Wiley \& Sons, New York-London-Sydney, 1978.

[26] J.-L. Lions, Quelques Méthodes de Résolution des Problèmes aux Limites Non Linéaires, Dunod Gauthier-Villars, Paris 1969.

[27] W. Liu, General decay and blow-up of solution for a quasilinear viscoelastic problem with nonlinear source, Nonl. Analysis 73 (2010) 1890-1904.

[28] W. Liu, Uniform decay of solutions for a quasilinear system of viscoelastic equations, Nonl. Analysis 71 (2009) 2257-2267. 
[29] A. E. H. Love, Treatise on Mathematical Theory of Elasticity, Dover Publications, New York, 1944.

[30] J. Málek \& D. Pražák, Large time behavior via the method of l-trajectories, J. Differential Equations 181 (2002) 243-279.

[31] S. A. Messaoudi, General decay of solutions of a viscoelastic equation, J. Math. Anal. Appl. 341 (2008) 1457-1467.

[32] S. A. Messaoudi \& N.-e. Tatar, Exponential and polynomial decay for a quasilinear viscoelastic equation, Nonl. Analysis 68 (2008) 785-793.

[33] S. A. Messaoudi \& N.-e. Tatar, Exponential decay for a quasilinear viscoelastic equation, Math. Nachr. 282 (2009) 1443-1450.

[34] S. A. Messaoudi \& N.-e. Tatar, Global existence and uniform stability of solutions for a quasilinear viscoelastic problem, Math. Meth. Appl. Sci. 30 (2007) 665-680.

[35] A. Miranville \& S. Zelik, Handbook of Differential Equations, Evolutionary Equations, Volume 4, Chapter 3, C.M. Dafermos and M. Pokorny, Editors, Elsevier, 2008.

[36] J. E. Muñoz Rivera, Asymptotic behaviour in linear viscoelasticity, Quart. Appl. Math. 52 (1994) 628-648.

[37] J. Y. Park \& S. H. Park, General decay for quasilinear viscoelastic equations with nonlinear weak damping, J. Math. Phys. 50, article 083505 (2009).

[38] V. Pata \& A. Zucchi, Attractors for a damped hyperbolic equation with linear memory, Adv. Math. Sci. Appl. 11 (2001) 505-529.

[39] A. Pazy, Semigroups of Linear Operators and Applications to Partial Differential Equations, Applied Mathematical Sciences Vol. 44, Springer-Verlag, New York, 1983. 
[40] M. Renardy, W. J. Hrusa \& J. A. Nohel, Mathematical Problems in Viscoelasticity, Pitman Monographs and Surveys in Pure and Applied Mathematics 35, Longman, Harlow, 1987.

[41] J. Simon, Compact sets in the space $L^{p}(0, T ; B)$, Ann. Mat. Pura Appl. 146 (1987) $65-96$.

[42] R. Temam, Infinite-Dimensional Dynamical Systems in Mechanics and Physics, Applied Mathematical Sciences 68, Springer-Verlag, New York, 1988.

[43] S.-T. Wu, Arbitrary decays for a viscoelastic equation, Boundary Value Problems 2011 (2011):28.

[44] Yang Zhijian, Finite-dimensional attractors for the Kirchhoff models, J. Math. Phys. 51, article 092703 (2010) .

[45] Yang Zhijian, Finite-dimensional attractors for the Kirchhoff models with critical exponents, J. Math. Phys. 53, article 032702 (2012) .

[46] Yang Zhijian, Global attractors and their Hausdorff dimensions for a class of Kirchhoff models, J. Math. Phys. 51, article 032701 (2010) .

[47] Yang Zhijian, Longtime behavior for a nonlinear wave equation arising in elastoplastic flow, Math. Meth. Appl. Sci. 32 (2009) 1082-1104.

[48] Yang Zhijian \& Jin Baoxia, Global attractor for a class of Kirchhoff models, J. Math. Phys. 50, article 032701 (2009) .

[49] K. Yosida, Functional Analysis, 6th edition, Springer-Verlag, New York, 1980. 